Review Article

\title{
Bioactive Foods and Medicinal Plants for Cardiovascular Complications of Type II Diabetes: Current Clinical Evidence and Future Perspectives
}

\author{
Roodabeh Bahramsoltani $\left(\mathbb{D},{ }^{1,2}\right.$ Mohammad Hosein Farzaei $(\mathbb{D}),{ }^{3,4}$ Mahboobe Ram $\left(\mathbb{D},{ }^{5}\right.$ \\ Shekoufeh Nikfar $\left(\mathbb{B},{ }^{6,7}\right.$ and Roja Rahimi $\mathbb{I}^{1,6}$ \\ ${ }^{1}$ Department of Traditional Pharmacy, School of Persian Medicine, Tehran University of Medical Sciences, Tehran, Iran \\ ${ }^{2}$ PhytoPharmacology Interest Group (PPIG), Universal Scientific Education and Research Network (USERN), Tehran, Iran \\ ${ }^{3}$ Pharmaceutical Sciences Research Center, Health Institute, Kermanshah University of Medical Sciences, Kermanshah, Iran \\ ${ }^{4}$ Medical Biology Research Center, Kermanshah University of Medical Sciences, Kermanshah, Iran \\ ${ }^{5}$ Student Research Committee, Faculty of Pharmacy, Mashhad University of Medical Sciences, Mashhad, Iran \\ ${ }^{6}$ Evidence-Based Medicine Group, Pharmaceutical Sciences Research Center, Tehran University of Medical Sciences, Tehran, Iran \\ ${ }^{7}$ Department of Pharmacoeconomics and Pharmaceutical Administration, Faculty of Pharmacy, \\ Tehran University of Medical Sciences, Tehran, Iran
}

Correspondence should be addressed to Roja Rahimi; rojarahimi@gmail.com

Received 3 November 2020; Accepted 1 September 2021; Published 16 September 2021

Academic Editor: Michał Tomczyk

Copyright (C) 2021 Roodabeh Bahramsoltani et al. This is an open access article distributed under the Creative Commons Attribution License, which permits unrestricted use, distribution, and reproduction in any medium, provided the original work is properly cited.

\begin{abstract}
Cardiovascular diseases (CVDs) are the main cause of mortality in type 2 diabetes mellitus (T2DM); however, not all patients are fully satisfied with the current available treatments. Medicinal plants have been globally investigated regarding their effect in CVD, yet the field is far from getting exhausted. The current paper aims to provide an evidence-based review on the clinically evaluated medicinal plants and their main therapeutic targets for the management of CVD in T2DM. Electronic databases including PubMed, Cochrane, Embase, Scopus, and Web of Science were searched from 2000 until November 2019, and related clinical studies were included. Lipid metabolism, glycemic status, systemic inflammation, blood pressure, endothelial function, oxidative stress, and anthropometric parameters are the key points regulated by medicinal plants in T2DM. Anti-inflammatory and antioxidant properties are the two most important mechanisms since inflammation and oxidative stress are the first steps triggering a domino of molecular pathological pathways leading to T2DM and, subsequently, CVD. Polyphenols with potent antioxidant and anti-inflammatory effects, essential oil-derived compounds with vasorelaxant properties, and fibers with demonstrated effects on obesity are the main categories of phytochemicals beneficial for CVD of T2DM. Some medicinal plants such as garlic (Allium sativum) and milk thistle (Silybum marianum) have strong evidences regarding their beneficial effects; however, others have low level of evidence which reveals the need for further clinical studies with larger sample sizes and longer follow-up periods to confirm the safety and efficacy of medicinal plants for the management of CVD in T2DM.
\end{abstract}

\section{Introduction}

Type 2 diabetes mellitus (T2DM) is a metabolic disorder accompanied with reduced glucose uptake, abnormal glucose metabolism, and insulin resistance which affects 1 in 11 adults [1]. Tracing the increasing rate of T2DM during the past decades shows its incidence and prevalence to be dramatically higher than the predicted values $[2,3]$.
T2DM is a principal risk factor for a broad spectrum of severe conditions amongst which the most important ones are cardiovascular diseases (CVDs) [4]. More than $80 \%$ of deaths in T2DM patients occur due to cardiovascular events which caused CVD to be the leading cause of mortality in these patients [5]. Based on the Framingham study, diabetic patients are two to six times more prone to myocardial infarction and heart failure [6]. A recent meta-analysis 
demonstrated higher risk of atrial fibrillation in diabetic patients [7]. Another meta-analysis revealed that diabetes significantly increases the risk of sudden cardiac death [8]. Also, hypertension is two times more prevalent in T2DM patients [9]. Diabetes is also an important risk factor for chronic heart failure [10]. Also, some cardiovascular drugs, such as statins, can increase the risk of diabetes[11]. Thus, there is a close relationship between T2DM and CVD.

Studies revealed that despite some beneficial effects of antihyperglycemic agents for the reduction of CVD risk [4], hyperglycemia is not the key concern in regard to CVD in T2DM patients [12]. In addition to glycemic control, specific management of CVD, e.g., regulation of lipid profile and HTN, is of a great importance [12]. In other words, antidiabetic agents such as metformin and glibenclamaide which only affect the blood glucose level does not have a considerable effect on CVD risk, whereas oral antidiabetics such as thiazolidinediones with insulin-sensitizing effects are more probable to reduce CVD risk $[12,13]$. On the other hand, thiazolidinediones are associated with side effects such as higher risk of bone fracture due to the suppression of proosteoblastic pathways [14]. Other drug choices for the management of CVD risk in T2DM patients include antiplatelet agents and anticoagulants [4]; however, their long-term efficacy is still in doubt and more important, the adverse effects can be disturbing in long-term use, causing low patient adherence and compliance. So, scientists are globally seeking new options with better efficacy and fewer side effects for the management of CVD in T2DM.

Medicinal plants have an ancient history of use amongst people which is currently known as complementary and alternative therapies $[15,16]$. Additionally, current scientific evidence supports the beneficial effects of herbal extracts and their isolated compounds in different types of CVD such as atherosclerosis [17], hypertension, and hyperlipidemia [18]. In diabetes-associated CVD, plant-derived natural supplements could play a positive role in preventing oxidative damage and inflammation [19] which are two basic mechanisms involved in the pathophysiology of T2DMassociated CVD. Thus, plants can be future candidates for the management of CVD in T2DM. The aim of the present study is to review controlled clinical trials on the cardiovascular effects of plants in T2DM patients.

\section{Methods}

2.1. Search Strategy. Electronic databases including Medline, Scopus, Embase, Web of Science, and Cochrane library were searched with the following search formula:

"diabetes" [title/abstract/keyword] AND "plant" OR "extract" OR "herb" [all fields] AND "cardiovascular" OR "atherosclerosis" OR "hypertension" OR "hyperlipidemia” OR "dyslipidemia” [title/abstract/keyword]

Articles were collected from January 2000 until November 2019. Primary search results were screened by two independent investigators. No language restriction was considered.
2.2. Inclusion and Exclusion Criteria. Inclusion criteria were controlled clinical trials (using placebo or no intervention design) in which the effect of a plant in regard to a cardiovascular parameter (lipid profile, blood pressure, endothelial dysfunction, oxidative stress, or systemic inflammation) was evaluated in T2DM patients. Exclusion criteria were animal and cellular studies, human studies other than clinical trials (e.g., cohort studies and case reports), comparing the results of the herbal intervention with a standard drug (in case the standard drug was administered to both test and control groups, the study was included), choosing healthy individuals as control subjects, including type 1 diabetic patients or patients with obesity or metabolic syndrome without a diagnosis of T2DM, and administration of a mixture of herbal and nonherbal materials. Studies that assessed the effect of herbal mixtures were also excluded because the results of those studies cannot be attributed to any of the individual herbal extracts. Also, purified phytochemicals were excluded since the aim of this review is to only consider plants since they are better choices to be suggested as dietary interventions. Studies on the antidiabetic activity of plant extracts without considering a cardiovascular parameter were excluded, as well. References of the included articles were also checked to find further relevant studies.

Final included papers were screened to extract the scientific name of the plant (in case the scientific name was not mentioned, the most probable scientific name was written in the table with an asterisk sign), used part, dosage, study design, sample size (the number of patients who completed the study), duration of treatment, and outcomes. Jadad score was used to evaluate the quality of the studies [20].

\section{Mechanisms of Plants Clinically Investigated to Control CVD in T2DM}

Among a total of 10644 primarily obtained papers, 73 were finally included. Figure 1 shows the detailed study selection process. Final included papers are summarized in Table 1. Medicinal plants have demonstrated several benefits to control cardiovascular complications of T2DM via different mechanisms. The most important medicinal plants exerting each mechanism are discussed as follows. Figure 2 shows a schema of the mechanisms affected by bioactive foods and plants to control CVD complications of T2DM.

3.1. Glycemic Profile. Despite the numerous large-scale clinical trials designed to clarify the relationship between blood sugar and CVD in T2DM patients, the problem is not yet completely solved since intensive blood glucose control has represented conflicting results [93]. Persistent hyperglycemia in diabetic patients leads to impaired angiogenesis which is, at least in part, related to abnormal glucose flux via the hexosamine biosynthetic pathway and participates in cardiovascular mortality [94]. Also, high level of blood glucose induces the production of advanced glycemic endproducts (AGEs) which accumulate during the time and cause vascular complications of T2DM [95]. Blood insulin 


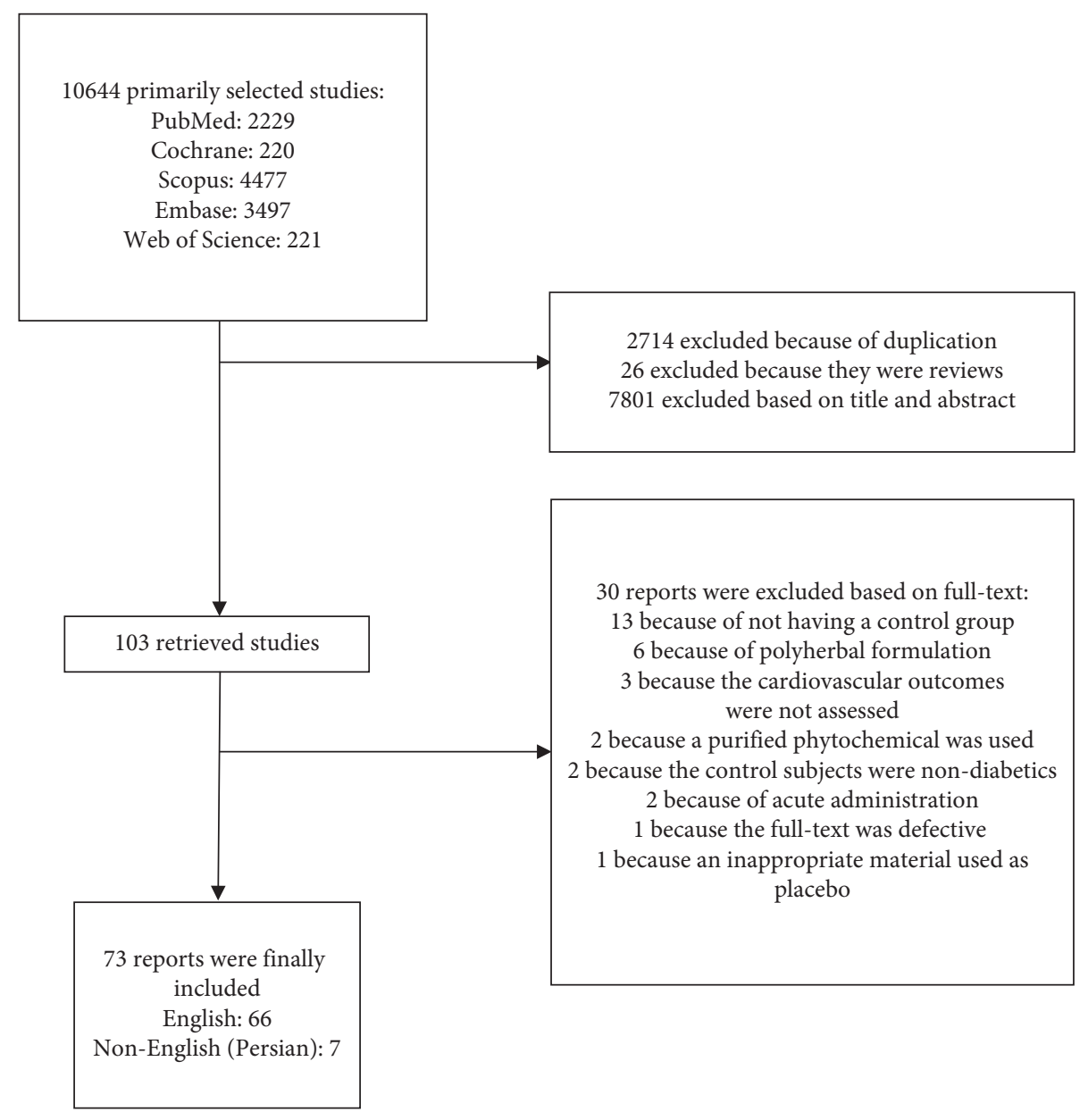

FIgURE 1: Study selection flow diagram.

level is another key factor launching the cardiovascular complications of T2DM. It is demonstrated that type 1 diabetic patients (in whom the main contributor is the impaired insulin level) have also high susceptibility to CVD. Thus, regardless of the other factors, abnormal blood glucose and insulin level themselves can be triggers for cardiovascular events. Insulin acts as a double-edged sword in the pathogenesis of CVD. In healthy subjects, insulin secretion leads to the dual activation of the mitogen-activated protein kinase (MAPK) and the phosphatidylinositol 3-kinase $(\mathrm{PI} 3 \mathrm{~K})$. While the former activates proatherogenic factors, the latter elevates nitric oxide (NO) production by endothelial nitric oxide synthase (eNOS) which causes a relaxing effect on the vascular smooth muscles and suppresses the proatherogenic and proinflammatory mediators in plasma. By contrast, during insulin resistance, the proatherogenic cascade is well activated, whereas the antiatherogenic pathway (PI3K) is not fully responsive [93].

Several medicinal plants have shown antihyperglycemic activity in clinical studies (Table 1). Milk thistle (Silybum marianum (L.) Gaertn.) is a well-known medicinal plant due to its ancient use as a hepatoprotective remedy; however, significant antioxidant properties of its flavonolignans made researchers reconsider its health benefits, and thus, it is now considered as an interesting option for the treatment of several chronic diseases involved with oxidative stress [96]. Six-week administration of milk thistle supplement to T2DM patients could significantly improve glycemic parameters via reduction of FBS, FPI, and insulin resistance which was evident from the homeostatic model assessmentinsulin resistance (HOMA-IR) and quantitative insulin sensitivity check index (QUICKI) [21]. The supplement also improved lipid profile and inflammation/oxidative damage biomarkers [22]; thus, it can be suggested as a valuable herb to manage CVD risk in T2DM [97]. Cynara scolymus L. or the globe artichoke is a popular vegetable widely used in different parts of the world. In a study by Nazni et al., five different food products containing artichoke extract were prepared in order to choose the most pleasant form for a clinical trial on the effect of dietary artichoke on cardiovascular parameters in T2DM patients. Wheat biscuit enriched with artichoke extract was chosen as the most acceptable form and was administered in a placebo-controlled trial to T2DM patients for a period of 90 days. The preparation could significantly improve both fasting and postprandial blood sugar, as well as the lipid profile [23]. Another trial also assessed the effect of globe artichoke in T2DM patients; however, two months of treatment resulted in no significant effect on glycemic parameters and TAG in this study and only reduced TC and LDL-C [24]. It should be 


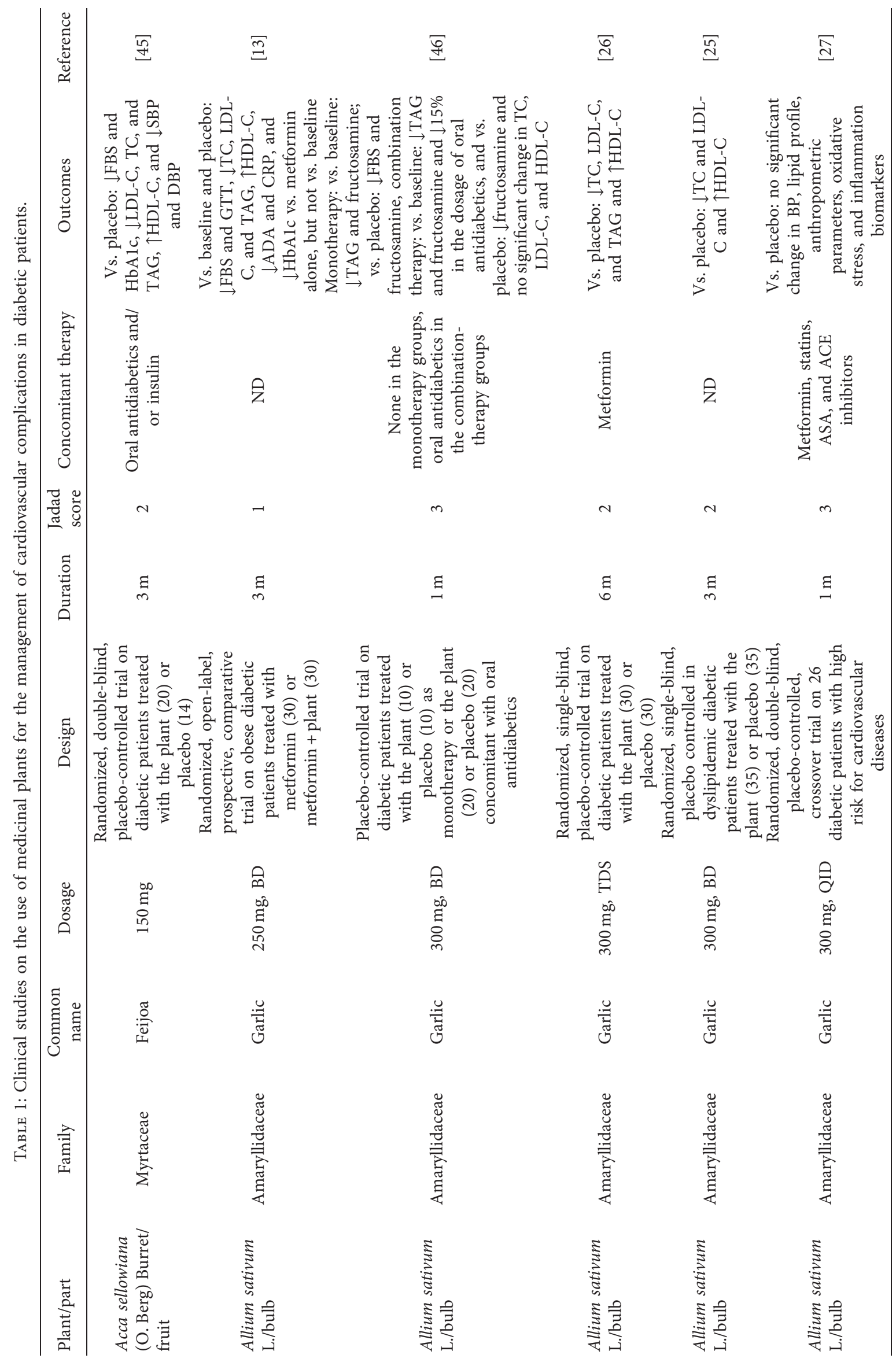




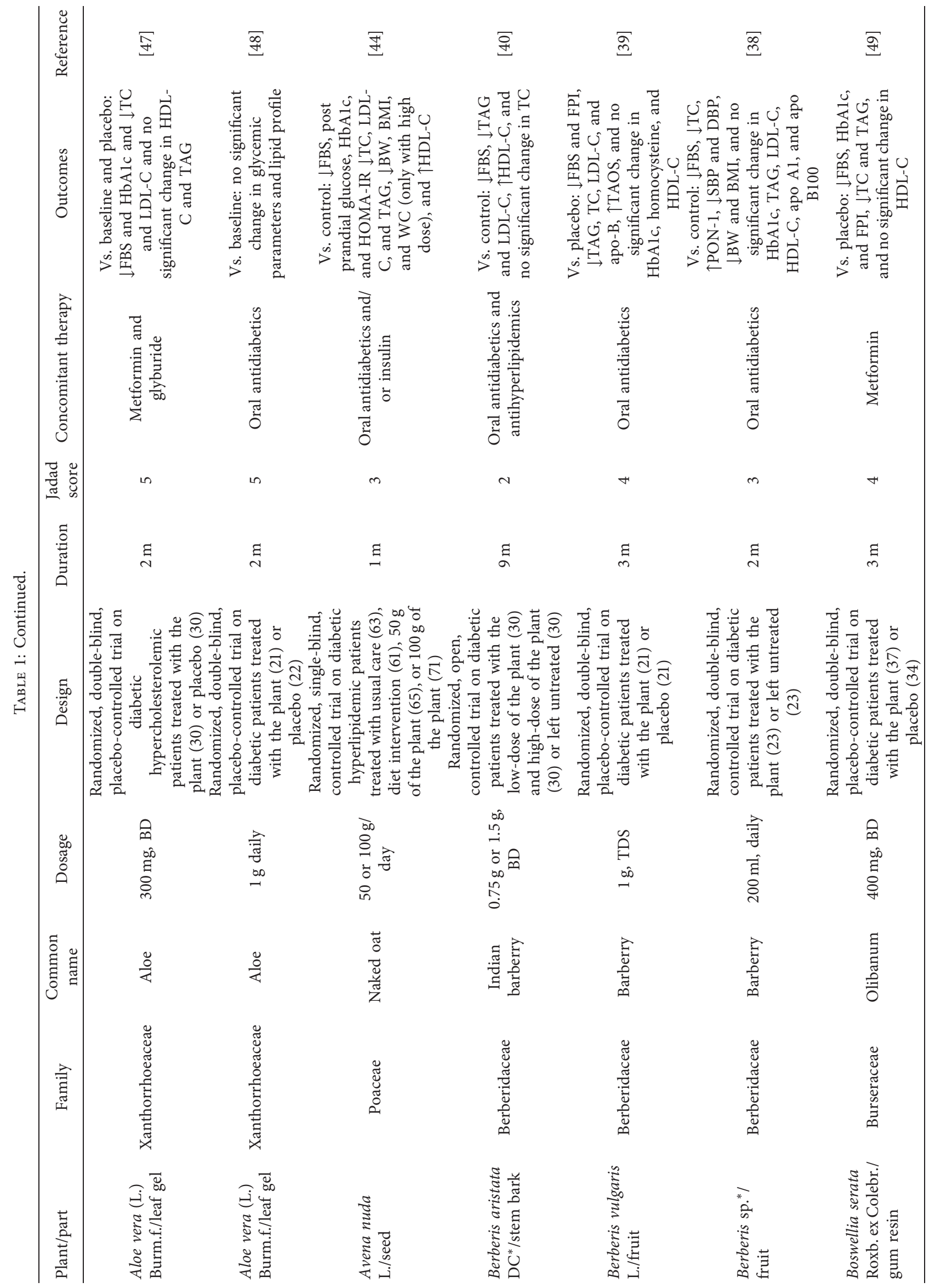




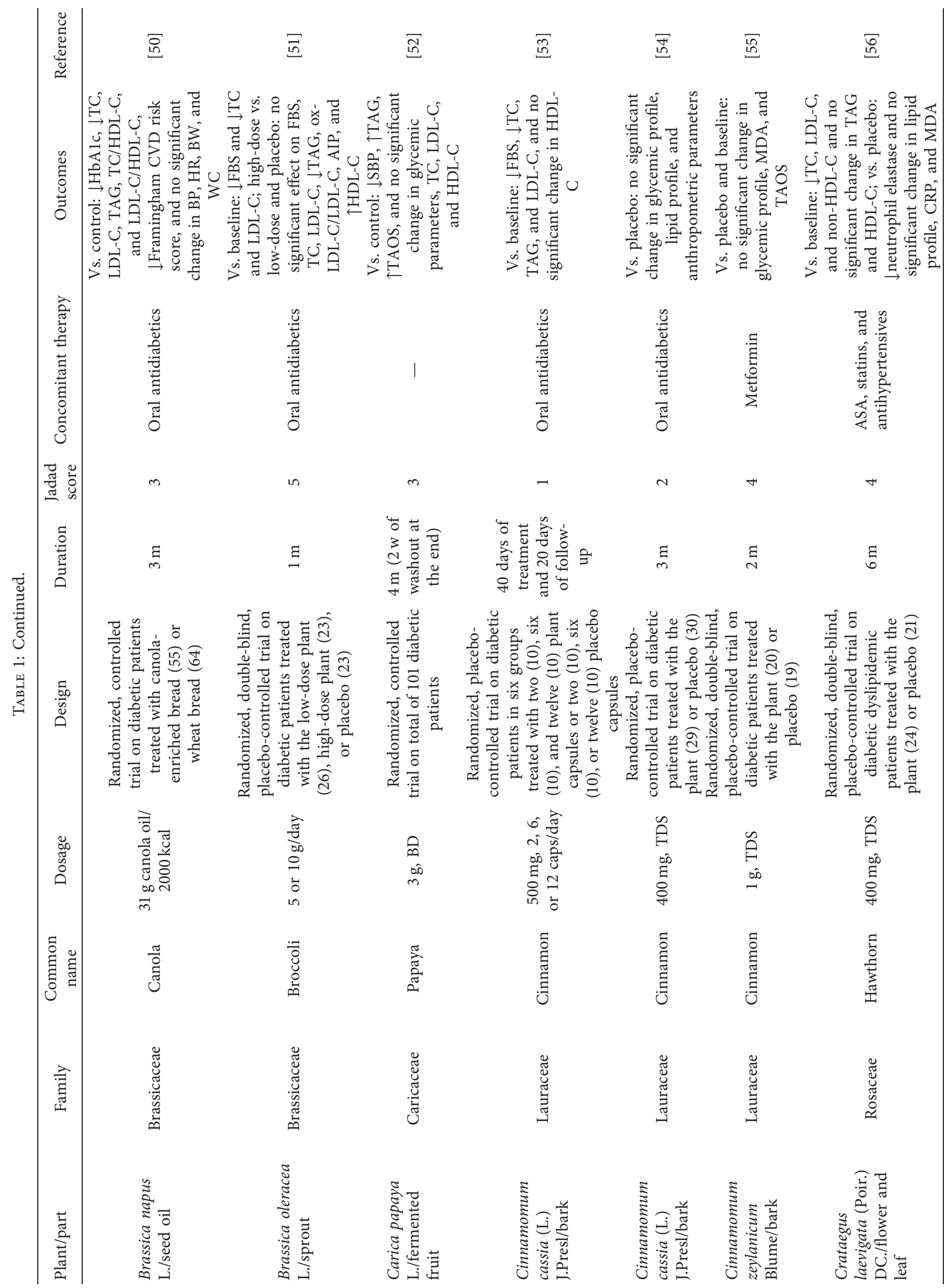




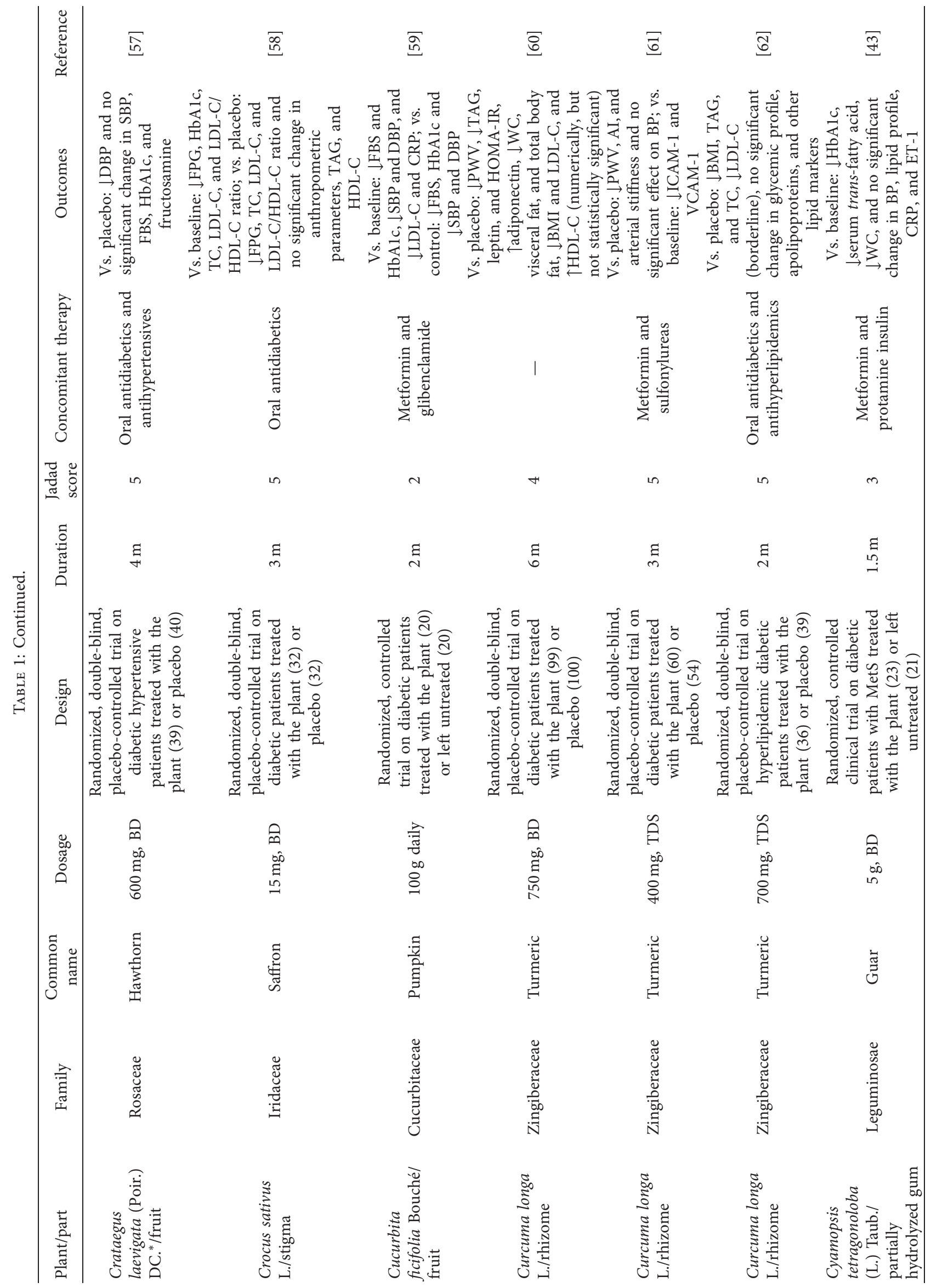




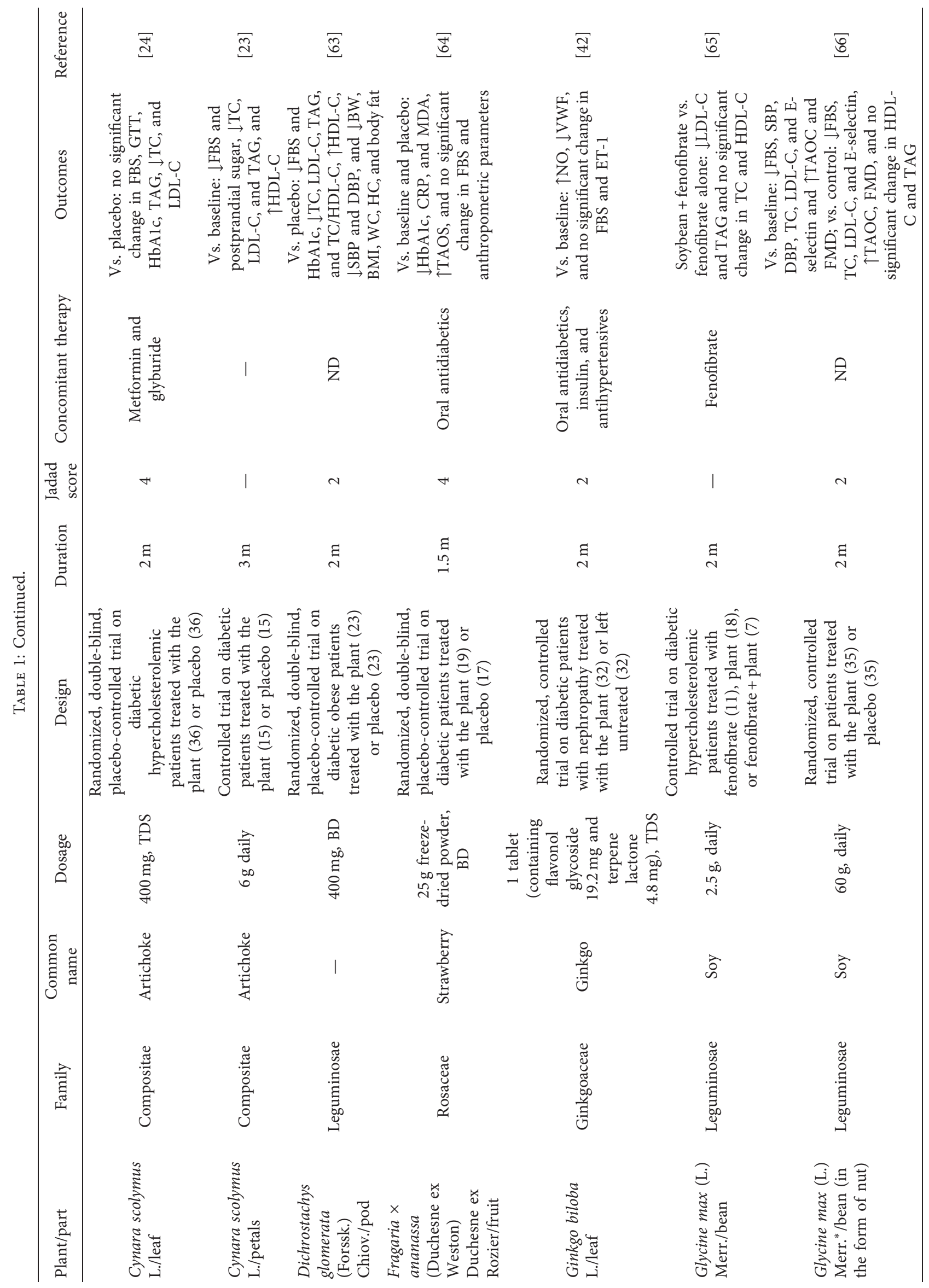




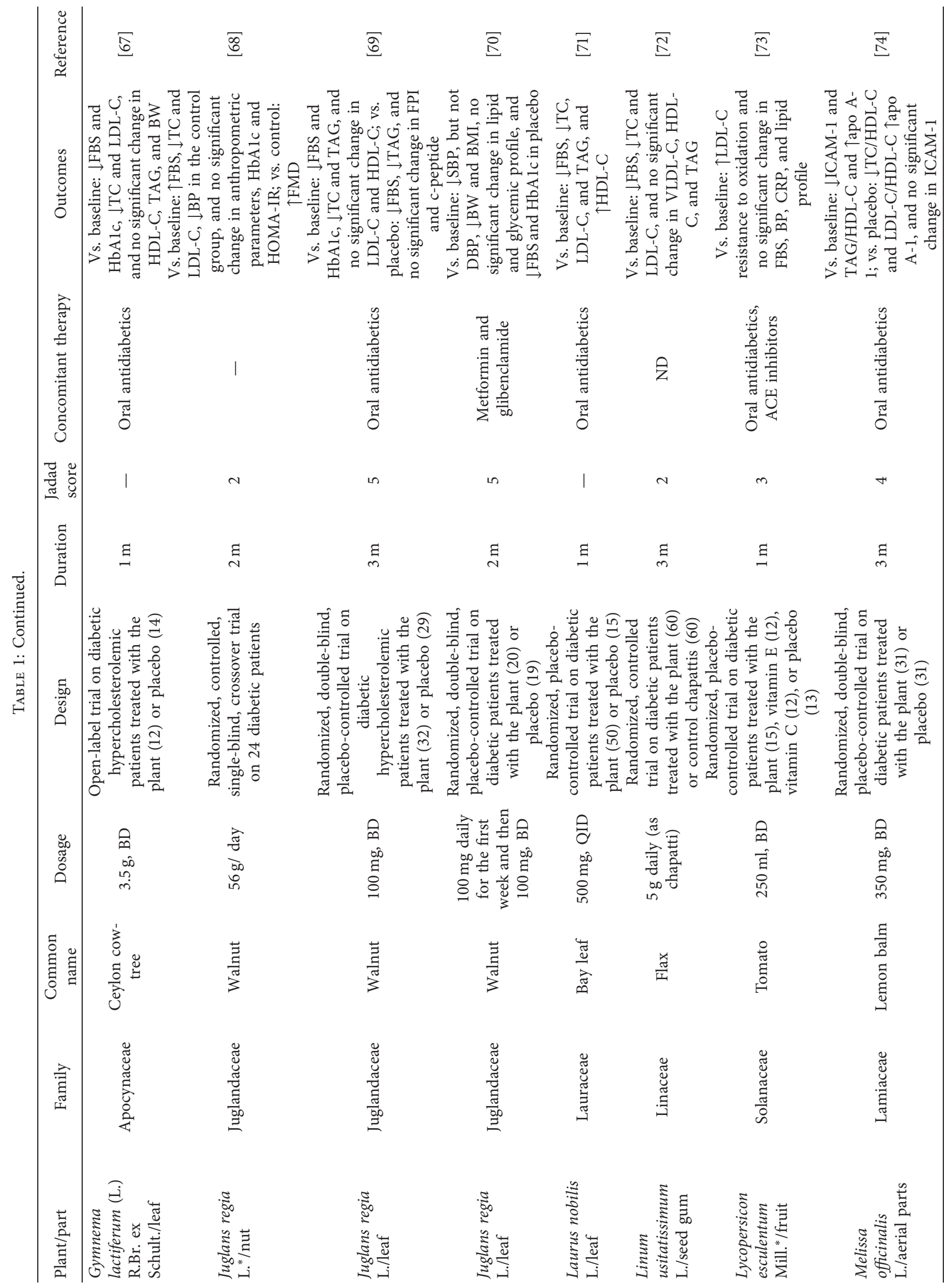




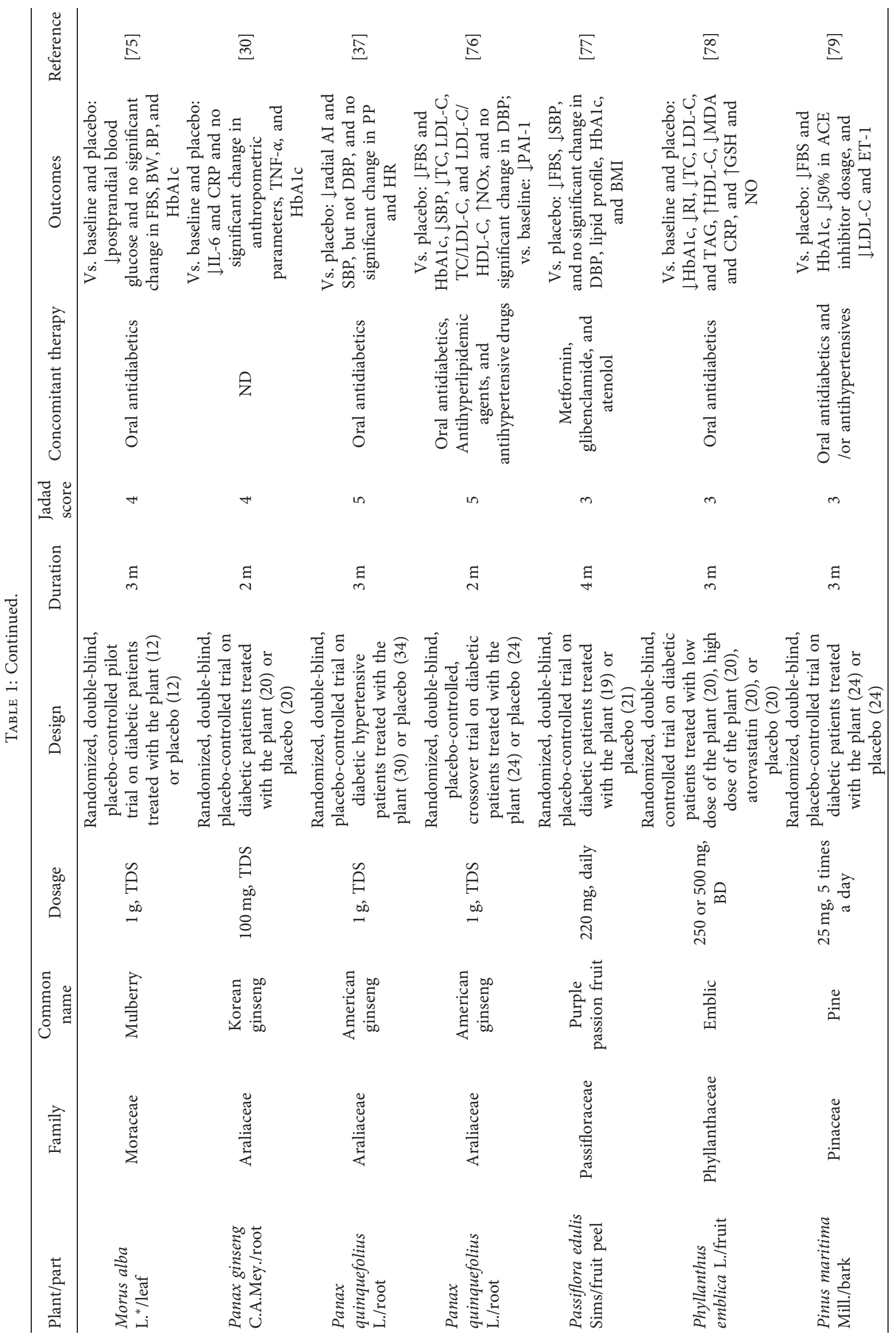




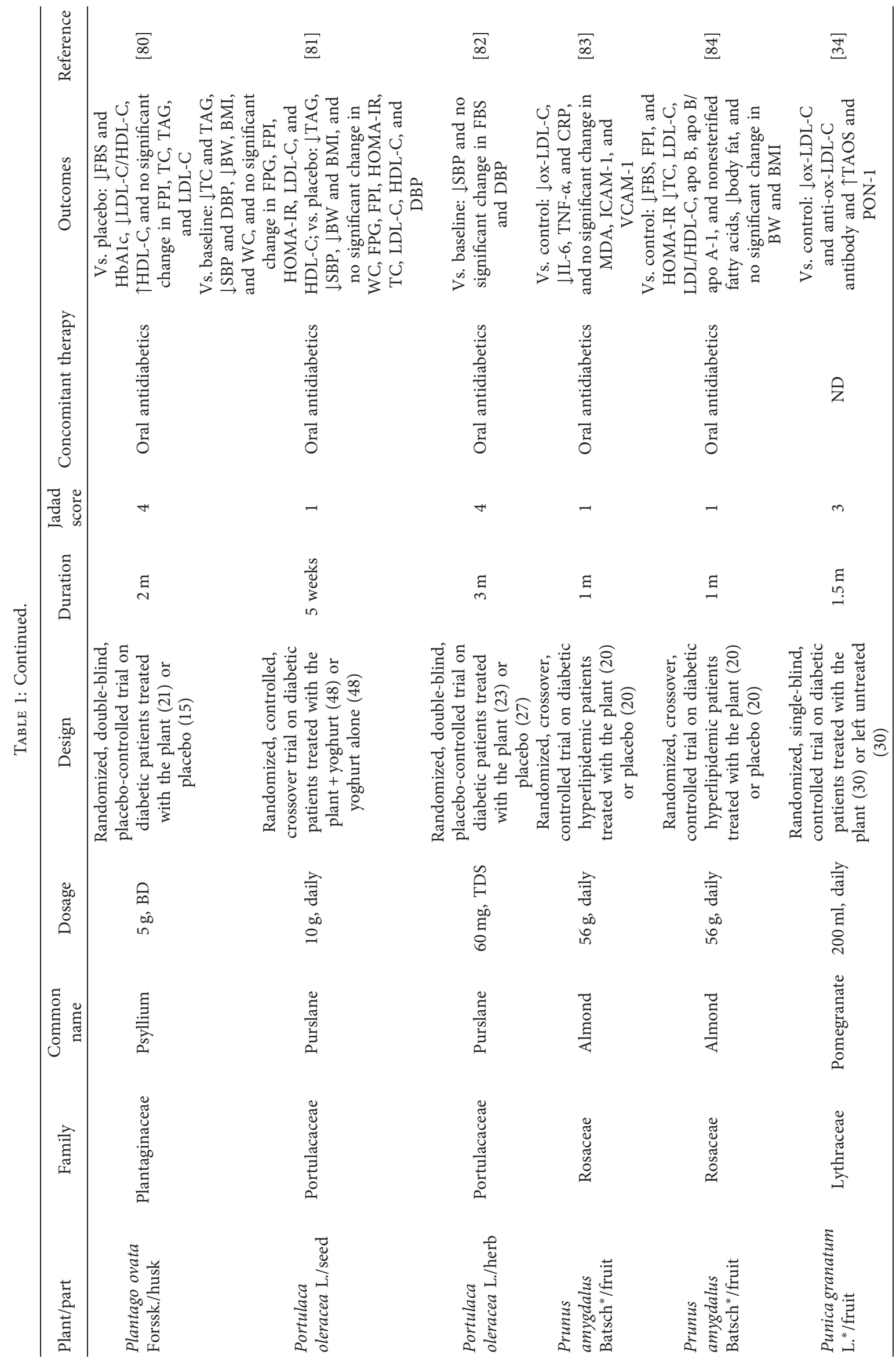




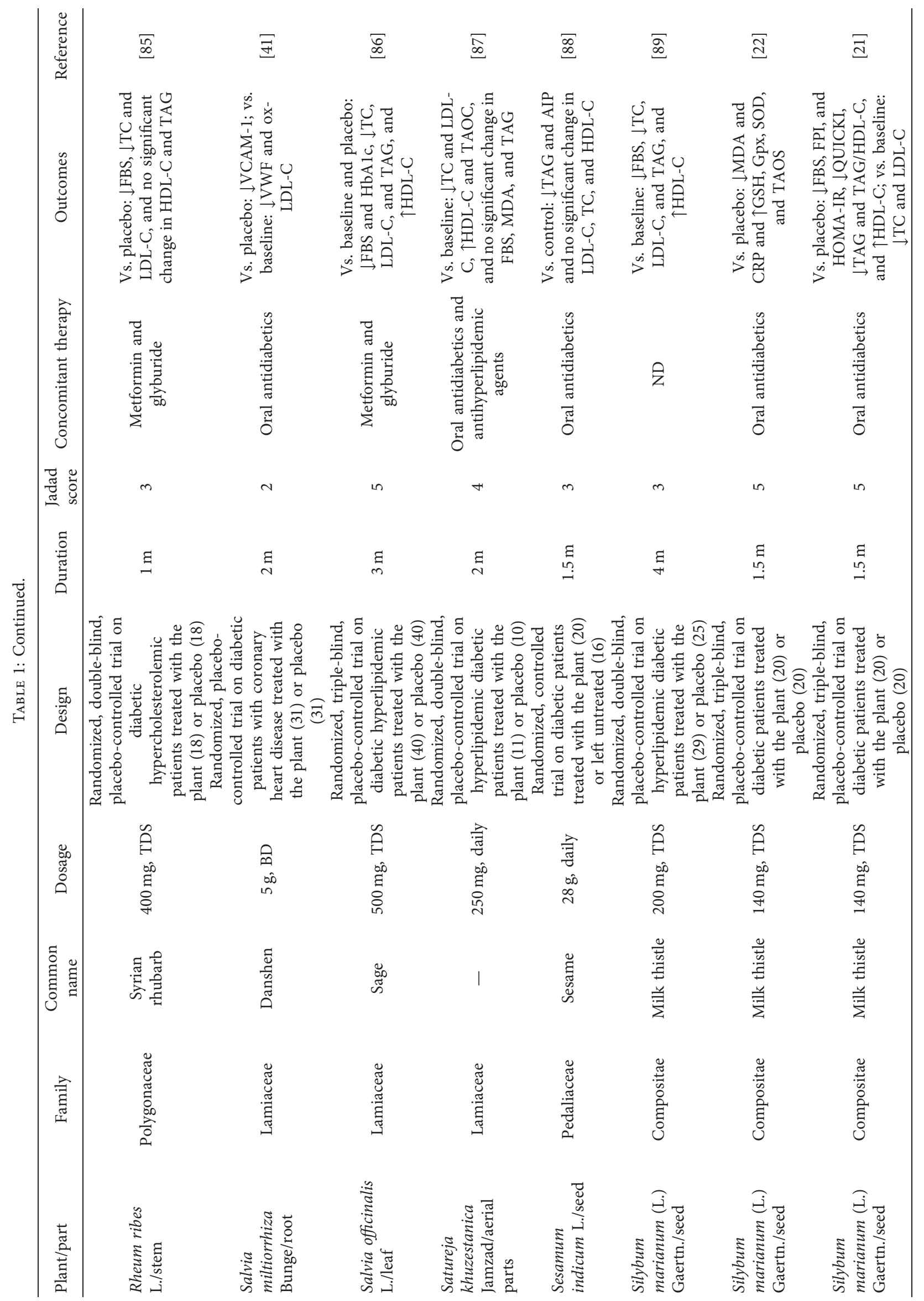




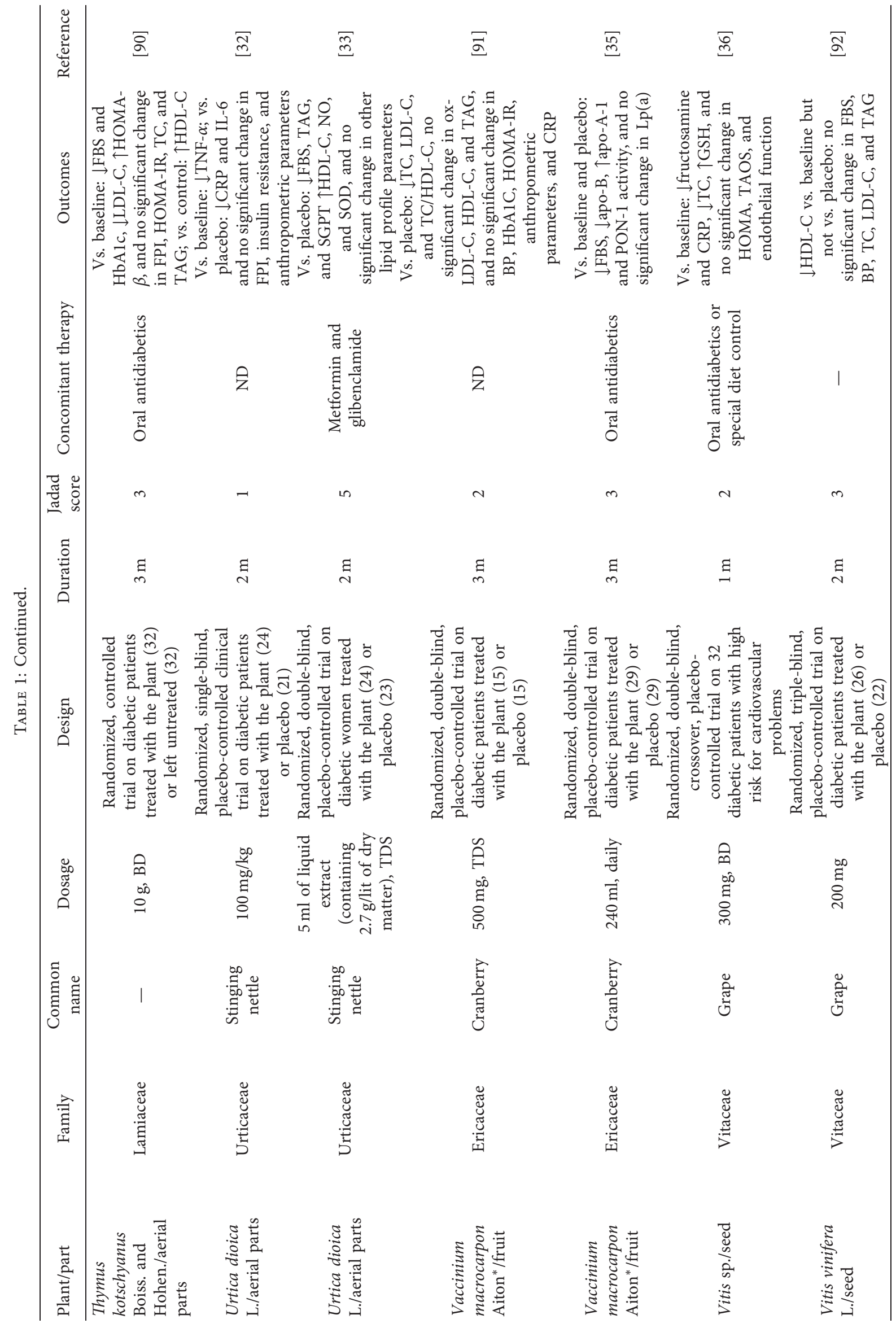




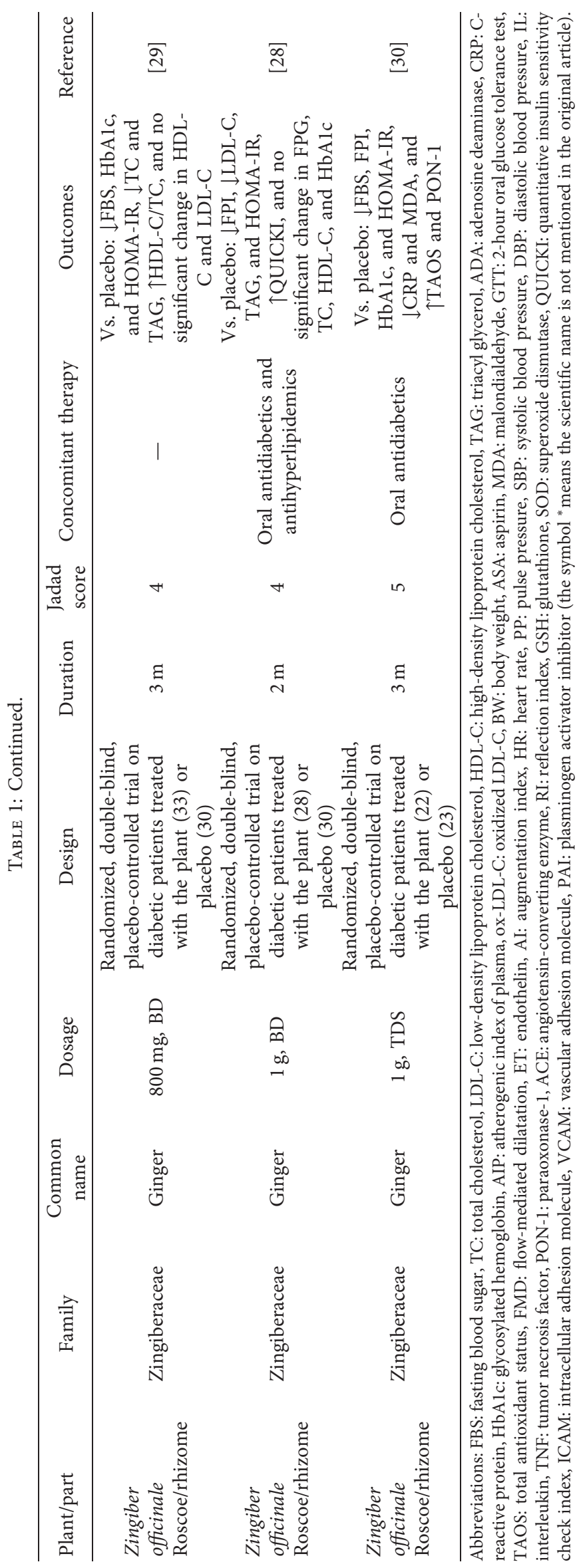




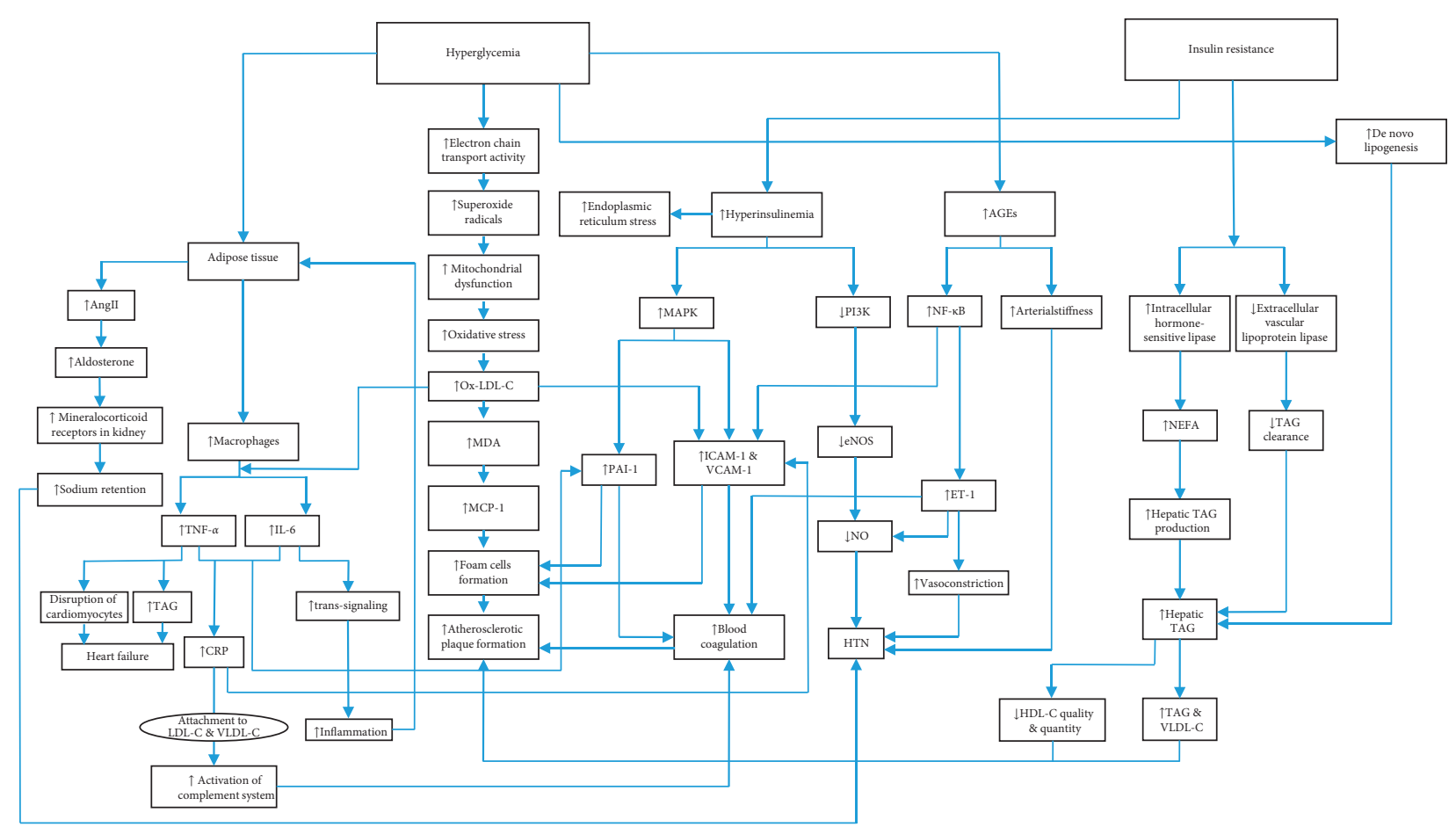

FIGURE 2: Therapeutic targets of bioactive foods and medicinal plants for the management of cardiovascular diseases in type 2 diabetic patients.

mentioned that, in addition to the shorter treatment period, the latter study used fiber-free extract, whereas the former study prepared the artichoke biscuits with the whole plant, containing fiber as well. This suggests the important role of dietary fibers in the clinical efficacy of artichoke.

3.2. Lipid Profile. Dyslipidemia is a common feature of T2DM with a prevalence of $37 \%$ to $56 \%$ [98]. The routine characteristics of diabetic dyslipidemia is hypertriglyceridemia in the form of elevated triacylglycerol-rich lipoproteins (TRLs), along with an increase in small dense low-density lipoprotein cholesterol (sdLDL-C) and a decrease in high-density lipoprotein cholesterol (HDL-C) [99]. TLRs are the result of fat digested from the foods as chylomicrons which contain apolipoprotein B48 (apoB48) or are released from the liver in the form of very-low-density lipoproteins (VLDL-C), containing apoB100 [100]. It seems that the reduction in CVD risk in diabetic patients via glycemic control is mostly due to the regulation of lipid profile rather than the glycemic profile itself [101]. Also, HDL-C is suggested to be an important factor which independently determines the risk of CVD in diabetic patients [102] since its quality and quantity are dysregulated even before the clinical diagnosis of diabetes [103]. In diabetic subjects, the level of intracellular hormone-sensitive lipase is augmented, while the extracellular vascular lipoprotein lipase is reduced due to insulin resistance or deficiency. The former results in the higher release of nonesterified fatty acids from adipose tissues which consequently increases hepatic production of triacylglycerol (TAG), whereas the latter causes reduced clearance of TAG from plasma; both finally result in the increased blood TAG level [103]. Increased blood sugar in T2DM elevates de novo lipogenesis from glucose [104]. Also, abnormal increase in insulin level along with insulin resistance causes disturbance in the production of hepatic sterols, endoplasmic reticulum stress, reduced apoB100 catabolism, and stimulation of VLDL-C production (especially $\mathrm{VLDL}_{1}$ which is larger and contains higher amount of TAG) that results in elevated blood VLDL$\mathrm{C}$ level [99]. On the other hand, abnormal structure of HDL$\mathrm{C}$, as well as a decrease in its production, causes the antiatherogenic activity of these particles to be reduced compared with that of normal subjects [99].

Considering the abovementioned importance of lipid levels in T2DM, it is not far-fetched that most studies assessing the effect of medicinal plants in T2DM evaluate the effect of the treatments on the lipid profile of patients. Garlic (Allium sativum L.) is amongst the most evident medicinal plants to regulate the lipid profile. The bulb contains sulfated compounds such as S-allyl cysteine, allicin, and alliin. When chopped, alliinase in garlic turns alliin to allicin [105]. Garlic is suggested to have an antihypertrophic effect on the heart via the elevation of hydrogen sulfide $\left(\mathrm{H}_{2} \mathrm{~S}\right)$ and NO [106]. Alliin has shown an inhibitory effect on 3-hydroxy 3-methyl glutaryl coenzyme A (HMG-CoA) reductase activity which is the same mechanism as statins [106]. Garlic also showed antihypertensive activity [107] as well as an inhibitory effect on adenosine deaminase (ADA) activity, an enzyme possibly involved in insulin resistance [108]. Some studies reported significant effect of garlic supplement with a dose of $500 \mathrm{mg} /$ day [108] or $300 \mathrm{mg}$, twice daily $[25,26]$ in the regulation of lipid profile; whereas some others such as the study of Atkin et al. reported no such effect even with a higher administered 
dose [27]. This might be due to the different design of the two studies as the patients in the latter study received conventional antidiabetics, as well. Also, the latter study assessed the effect of garlic for a shorter period of time which shows the need for longer treatment to achieve the therapeutic effect. Overall, garlic can be considered as an important dietary intervention to manage a series of cardiovascular complications such as HTN, hyperlipidemia, and atherosclerosis [109] which is also reported in T2DM patients; however, future studies are needed to determine the optimum dose and dosage form to achieve a satisfying therapeutic outcome. Ginger rhizome (Zingiber officinale Roscoe) is another medicinal plant which is also used as a popular spice in cooking. Three months of supplementation with ginger in T2DM patients could significantly decrease LDL-C and TAG and improve HDL-C. Ginger could also significantly reduce fasting plasma insulin and HOMA-IR, showing an overall improvement in lipid profile, as well as insulin resistance [28]. These results regarding the insulin resistance and TAG were also confirmed in another study by Arablou et al.; however, the reported data on the LDL-C, HDL-C, and FBS were somehow controversial [29]. While the former study reported a nonsignificant effect on blood glucose, HDL-C, and HbAlc, the latter study showed a significant decrease in FBS and $\mathrm{HbA1c}$, as well as an increase in HDL-C which might be due to the longer period of supplementation. Ginger could also improve the biomarkers of oxidative damage and inflammation [30], suggesting the plant as a multipotential choice to manage CVD in T2DM. Gingerol and shogaol are two main active ingredients of the plant responsible for several pharmacological activities such as anti-inflammatory and vasorelaxant effects via the inhibition of prostaglandin and leukotriene synthesis. Also, ginger has demonstrated antiplatelet activity in animal studies which is another beneficial mechanism in CVD [110]; however, it should be noted that, in patients taking conventional antiplatelet agents, high doses of ginger may show a synergistic pharmacodynamic herb-drug interaction, thus, should be taken with caution.

3.3. Systemic Inflammation. The presence of inflammation in T2DM has been demonstrated years ago through cohort studies that revealed higher incidence of the disease in patients with higher level of acute-phase proteins such as C-reactive protein (CRP) and proinflammatory cytokines such as interleukin-6 (IL-6) in comparison to the subjects with normal values of these markers [111]. The increased level of inflammatory markers in T2DM is suggested to be mostly due to a general inflammatory status involving the whole body's immune system rather than a local inflammation of pancreas. Adipose tissue, abnormally hosting a large number of macrophages in obese subjects which produce tumor necrosis factor (TNF), and the liver, in which TNF- $\alpha$ - and IL-6-induced CRP is produced, seem to be the main participants in this process [112]. Inflammation is a common characteristic of T2DM and CVD. Overproduction of TNF- $\alpha$ and proinflammatory ILs results in cardiovascular events such as atherosclerosis. The importance of CRP in the prediction of CVD risk in T2DM is as high as LDL-C and HDL-C. On one hand, CRP attaches to LDL-C and VLDL-C particles and triggers blood coagulation via the activation of the complement system. On the other hand, it stimulates the production of soluble adhesion molecules, facilitating the formation of foam cells, and makes the endothelium of blood vessels prone to atherosclerotic plaque formation [113]. The deep involvement of CRP in cardiovascular events, along with its long half-life suggests this marker as an independent factor predicting CVD risk in T2DM [114]. Elevated level of TNF- $\alpha$ is also associated with increased risk of heart failure via the elevation of TAG, possibly due to stimulating VLDL-C production and disruption of cardiomyocytes [113]. In contrast, IL-6 seems to have a pleiotropic role in CVD which in not yet completely clarified; however, the mechanisms are somehow explained after the discovery of its two underlying signaling pathways, i.e., classic signaling and trans-signaling. While the activation of the former pathway results in anti-inflammatory effects and regulates the metabolism, the latter exacerbates inflammation during pathological conditions. The ratio of IL-6, soluble IL-6 receptor, and glycoprotein 130 (gp 130, a common signal transducer in the IL-6 family) determines the final results of IL-6 activation [115]. It is also demonstrated that IL- 6 and TNF- $\alpha$ interaction plays an important role in endothelial dysfunction in the animal model of diabetes [116].

Some medicinal plants have been reported to be effective on the serum level of inflammatory markers in T2DM patients. Panax ginseng C.A. Mey. (Chinese ginseng or Korean ginseng) is a globally well-known medicinal plant with a wide spectrum of indications in traditional Chinese medicine (TCM). Administration of ginseng supplement to diabetic patients over a period of eight weeks significantly reduced the serum level of IL-6 and CRP in comparison to both baseline values and the placebo group [31]. One of the main classes of active ingredients of ginseng are triterpene structures called ginsenoside, several of which have demonstrated anti-inflammatory properties via the modulation of IL-6 production [117], and their beneficial effects in CVD are demonstrated in preclinical studies [118]. Another medicinal plant effective on the level of inflammatory markers is stinging nettle (Urtica dioica L.) which decreased IL- 6 and CRP vs. placebo and TNF- $\alpha$ compared with baseline levels [32]. Another trial also demonstrated a higher level of NO in patients treated with nettle extract in comparison to placebo [33]. Based on the preclinical investigations, the antidiabetic effect of stinging nettle is attributed to its phenolic compounds and seems to be due to cytoprotective effects on the pancreas, $\alpha$-glucosidase and $\alpha$-amylase inhibition, and modulation of glucose transporter type 4 [119]; however, the exact subcellular mechanism of the plant in the modulation of inflammatory mediators needs to be further investigated.

3.4. Oxidative Stress. Oxidative stress is an inseparable part of T2DM and is closely related to the cardiovascular complications of this disease $[120,121]$. High blood sugar 
causes oxidative stress via the elevation of AGEs, glucose auto-oxidation, and hexosamine and polyol pathways induction, as well as mitochondrial damage due to overactivation of the electron chain transport system, resulting in the overproduction of superoxide radicals which are naturally deactivated by superoxide dismutase (SOD) enzyme [95, 122]; however, the excessively produced radicals directly destruct mitochondrial DNA. This damage causes a series of events leading to mitochondrial dysfunction and abnormal cellular energy expenditure, further inducing oxidative stress [95]. Oxidative stress in diabetic patients causes LDL-C particles turn into an oxidized form (OxLDL-C), evident from the serum level of malondialdehyde (MDA, a byproduct of lipid peroxidation) that consequently stimulates the production of monocyte chemoattractant protein-1 (MCP-1), a trigger for the formation of foam cells [121]. This oxidation process is partially prevented by paraoxonase-1 (PON-1), an esterase linked with apo A-1 of HDL-C particles, thus, plays a protective role against the development of atherosclerotic plaque formation [123]. PON-1 is also suggested to be a reliable marker for the prediction of atherosclerosis risk in T2DM [124]. Glutahtoine (GSH) is another part of the endogenous antioxidant defense mechanism which is altered in T2DM. It is demonstrated that the GSH level of erythrocytes and its absolute synthesis rate in diabetic patients are significantly lower than those of normal subjects, possibly due to nonglycemic mechanisms [125].

Punica granatum L. (pomegranate) is native to the Mediterranean region, especially Iran. The fruit juice is a rich source of two polyphenol subcategories, namely, anthocyanins and ellagitannins, with potent antioxidant and anti-inflammatory properties, as well as previously demonstrated beneficial effects in cardiovascular problems [126]. In a randomized, single-blind clinical trial, T2DM patients received a daily amount of $200 \mathrm{ml}$ pomegranate juice for 6 weeks. Compared to the untreated group, pomegranate could significantly improve antioxidant status via the elevation of PON-1 and decrease in Ox-LDL-C, as well as its specific antibodies [34]. Shidfar et al. reported the significant effect of cranberry, another anthocyaninrich fruit, toward the prevention of oxidative damage in T2DM via the improvement of PON-1 [35]. Grape seed extract, rich in proanthocyanidins, has demonstrated antioxidant properties in T2DM which was evident from the increased level of GSH [36]. It seems that anthocyanin-rich fruits are a reliable source of antioxidant components, especially in regard to improvement in PON-1 activity, thus, are clinically valuable to manage chronic oxidative damage in T2DM [127].

3.5. Blood Pressure. Hypertension (HTN) in T2DM is linked with a dramatic raise in the incidence of CVD so that a $10 \mathrm{mmHg}$ decrease in systolic blood pressure (SBP) is accompanied with $11 \%$ and $13 \%$ lower risk of myocardial infarction and microvascular complications, respectively [128]. Also, T2DM patients with diastolic blood pressure (DBP) lower than $80 \mathrm{mmHg}$ showed a lower risk of stroke and mortality compared with those with a DBP of $90 \mathrm{mmHg}$ [129]. Chronic inflammation in T2DM causes endothelial dysfunction, evident from the reduction of flow-mediated dilatation (FMD), makeing an imbalance between endogenous vasodilators such as $\mathrm{NO}$ and vasoconstrictors such as endothelin-1 (ET-1). Also, inflammation and oxidative stress stimulate the production of antgiotensin II in adipose tissue which results in increased aldosterone, the activation of mineralocorticoid receptors in the kidney, sodium retention, and consequently, HTN $[130,131]$. Furthermore, the production of AGEs due to high blood sugar causes stiffness of the arterial wall, further exacerbating HTN [9].

American ginseng (Panax quinquefolius L.) is grown in the North American continent. In a clinical trial in T2DM patients with well-controlled essential HTN, American ginseng ethanolic extract, standardized based on $10 \%$ of total ginsenosides, was administered to evaluate the vascular effects. The results showed a significantly lower SBP in the active group compared with placebo. Additionally, the augmentation index which is a noninvasive method for the evaluation of arterial stiffness was significantly reduced in patients treated with ginseng [37]. Preclinical studies suggest the inhibitory effect on glucotoxicity and endothelial dysfunction [132] and vascular smooth muscle cell proliferation [133] to be the possible vasculoprotective mechanisms of American ginseng. Barberry is another plant that demonstrated beneficial effects on both SBP and DBP in T2DM patients compared with the no intervention group [38]. This effect may be partly mediated by berberine, an alkaloid in barberry fruit, with previously demonstrated antihypertensive and vasodilatory properties in preclinical studies $[134,135]$. Barberry fruit was also effective in reducing blood glucose and insulin, as well as lipid profile, which is also attributed to berberine [39]. Barberry fruit contains anthocyanins, a group of polyphenols, which may also contribute to the health benefits of the fruit; however, a clinical trial on the barberry stems containing $2.23 \%$ berberine as the major alkaloid [40] (but a possibly lower anthocyanin content) in T2DM patients further confirms the significant role of this alkaloid in glycemic and cardiovascular effects of barberry.

3.6. Endothelial Function. Endothelial dysfunction is suggested as an independent predictor of T2DM development risk. Adhesion molecules including intracellular adhesion molecule (ICAM-1) and vascular adhesion molecule-1 (VCAM-1) are produced as a result of endothelial dysfunction. They are demonstrated to get increased years before the diagnosis of T2DM, and thus, they are not only the markers of vascular damage but also predict the occurrence of T2DM $[136,137]$. High production of AGEs in T2DM activates nuclear factor- $\kappa \mathrm{B}(\mathrm{NF}-\kappa \mathrm{B})$ which consequently stimulates gene expression of several factors, including ICAM-1, VCAM-1, and ET-1 [138]. Enhanced activity of ET-1 negatively affects the cardiovascular status of diabetic patients due to vasoconstrictor and procoagulant activities of ET-1, as well as a reduction in NO-mediated 
vasodilation [139]. On the other hand, proinflammatory cytokines such as TNF- $\alpha$ and IL- 6 trigger the coagulation process via the elevation of von Willebrand Factor (VWF) and plasminogen activator inhibitor-1 (PAI-1), resulting in a procoagulant state in the impaired endothelium. Furthermore, increased ox-LDL-C induces the production of proinflammatory cytokines, MCP-1, and adhesion molecules, all of which facilitate the process of atherosclerosis [138].

In a pilot-controlled trial, Salvia miltiorrhiza Bunge. was assessed in T2DM patients with coronary heart disease. The plant is commonly known as danshen and is a popular natural remedy for CVD in TCM. Two-month supplementation with danshen extract significantly decreased the level of VCAM-1 and VWF, demonstrating an improvement in endothelial function [41]. Salvianolic acids and diterpene structures called tanshinones are two major categories of danshen phytochemicals, both of which participate in beneficial effects of the plant in CVD [140]. Another study evaluated the vasculoprotective effect of ginkgo (Ginkgo biloba L.) on T2DM patients with nephropathy. Ginkgo, known as a living fossil, contains a mixture of flavonoids (ginkgoflavone glycosides) and diterpenes (ginkgolides and bilobalide) as the main active ingredients [141]. The plant has shown an inhibitory effect on ox-LDL-C-induced endothelial dysfunction in human umbilical vein endothelial cells [141]. Supplementation with ginkgo leaf extract could significantly attenuate the abnormally increased VWF and elevate NO level. Also, there were no significant differences in FBS, showing that the plant can be coadministered along with conventional antidiabetics without increasing the risk for hypoglycemia [42].

3.7. Anthropometric Parameters. The relationship between obesity and T2DM has been demonstrated in several studies. Recently published results of the ACCORD (Action to Control Cardiovascular Risk in Diabetes) study showed that T2DM patients with a body mass index (BMI) higher than 40 have the highest risk of nonfatal myocardial infarction and cardiac death [142]. Adipocytes secrete nonesterified fatty acids and hormones such as adiponectin and leptin which affect metabolism and insulin sensitivity. The imbalance between the release of these agents results in $\beta$-cell dysfunction and insulin resistance [143]. Molecular assessments also revealed that the gene expression of AGE ligands, NF- $\kappa \mathrm{B}$, and PI3K is dysregulated in obesity and T2DM [144]. Although obesity, in general, is known as a risk factor for T2DM, the distribution of body fat is a more important indicator of insulin resistance. It is suggested that waist circumference is the determinant of abdominal obesity and can be used to predict the risk of T2DM and CVD $[145,146]$. Aside direct exacerbating effects of T2DM on CVD, the coexistence of obesity in these patients further worsens the condition since an accumulating body of evidence introduces obesity as one of the prime suspects of CVD [147].

Guar gum is a product of Cyamopsis tetragonoloba (L.) Taub. seeds and is a water-soluble polymer of galactose and mannose usually used in a partially hydrolyzed form due to the unpleasantly high viscosity of the original form [148]. Six-week supplementation with daily $10 \mathrm{~g}$ dose of guar gum could significantly reduce $\mathrm{HbAlc}$ and serum transfatty acids compared with baseline values. The plant could also significantly reduce waist circumference; however, no such effect was observed for body weight (BW) [43]. Previous studies have shown beneficial effects of guar gum in weight loss due to the gel-forming properties and decreasing gastric emptying speed, as well as antiappetite activity [148]; thus, lack of the slimming effect in this trial might be due to the short study period or low administered dose. Another plant with high content of water-soluble fiber which is assessed in regard to CVD risk factors in T2DM is Avena nuda L., commonly known as naked oat. In this dietary intervention, patients were instructed to replace one of the main foods of their regimen with a product providing 50 or $100 \mathrm{~g}$ of naked oat. One month of naked oat intake decreased BW, BMI, and waist circumference in comparison to the control group in a dosedependent manner. Both lipid and glycemic profile were also improved during this intervention [44]. It can be inferred from the two studies that water-soluble fibers are one of the important categories of plant-based products able to help weight loss and glycemic/lipid profile regulation in T2DM patients; however, since they are mostly effective in high doses, they are suggested to be administered as dietary interventions.

\section{Safety}

Most of the studies included in this review reported no significant difference between the frequency of adverse effects in the active and passive groups, especially in case of dietary interventions, showing that the preparations were well tolerated. Most of the reported adverse effects were limited to transient low-grade gastrointestinal complications which disappeared after a while or by dose reduction. Also, some studies assessed the safety by measuring the biomarkers of hepatic and renal toxicity including aspartate transaminase (AST), alanine transaminase (ALT), and alkaline phosphatase (ALP), as well as blood urea and creatinine, respectively. Most studies reported no significant change in the evaluated biomarkers except within the normal range. Taken together, herbal interventions are generally safe in regard to the adverse effects; however, some other considerations such as the possibility of herbdrug interactions via pharmacokinetic or pharmacodynamic interactions with the conventional antihyperglycemic and cardiovascular drugs are possible which should be taken into account $[149,150]$. As an example, ginseng and garlic can increase prothrombin time, and if being administered to patients under treatment with aspirin or warfarin, the synergistic effect can result in abnormal bleeding [151]. Also, mucilaginous herbal materials, such as different gums, as well as resinous compounds, can delay/decrease the oral absorption of concomitantly used conventional drugs and consequently affect their pharmacokinetics; thus, it is recommended to 
take such herbal supplements with a proper time interval with conventional drugs [152].

\section{Discussion}

The current paper reviewed recent advances regarding the effectiveness of plants as dietary interventions for the management of cardiovascular complications in T2DM. It is well understood that any individual plant can act via several mechanisms which suggest it as a multifaceted approach to target different pathways involved in the pathogenesis of CVD in T2DM.

Two of the most important mechanisms by which several medicinal plants could improve cardiovascular outcomes of diabetic patients are antioxidant and anti-inflammatory properties. Oxidative damage and inflammation can trigger each other due to a negative feedback loop so that the free radicals can induce inflammation, and inflammatory mediators increase oxidative stress in a chronic pathological condition such as T2DM. Discovering detailed cellular pathways which participate in the pathogenesis of cardiovascular damage is the Rosetta stone for decoding the fundamental therapeutic targets in CVD of T2DM patients. Plants are rich sources of secondary metabolites preventing the free radicals produced due to the oxidative damage in different tissues which is now demonstrated in clinical studies (Table 1). Although most of the included trials have focused on the lipid and glycemic profile which are classic outcomes to be assessed in CVD, recent studies have tried to also measure oxidative damage and inflammation biomarkers due to the growing evidence supporting the involvement of these two mechanisms in the primary stages of T2DM.

The most important category of plant-derived secondary metabolites with antioxidant activities are polyphenols (Table 2). Polyphenols comprise several subcategories including flavonoids, anthocyanins, lignans, and phenolic acid, each of which have numerous number of studies supporting their antioxidant and anti-inflammatory activities [17]. Curcumin, resveratrol, quercetin, and epigallocatechin gallate (EGCG) which are today widely used as antioxidant supplements all belong to different classes of polyphenols.

Another important category of phytochemicals are essential oil-derived compounds such as small terpenes and terpene alcohols. These compounds have demonstrated relaxing effects on the muscle cells, thus, can improve HTN via vasorelaxant effects on the smooth muscles of blood vessels $[153,154]$.

Fiber-containing plants are also significantly effective in controlling CVD risk factors in T2DM patients by slowing the absorption of dietary fats and improvement of anthropometric parameters. One of the positive points regarding this category of phytochemicals is that they are usually tasteless compounds, so they can be prepared as different enriched food products without affecting the original taste, providing higher patient compliance.

In addition to fibers, other herbal materials can also be provided as enriched foods, e.g., beverages, breads, biscuits, corn flakes, or other types of usually taken snakes. Plants with active ingredients resistant to heat can be added to baked products such as the preparation used in the study of Nazni et al. [23]. Anthocyanin-rich fruits such as barberry, pomegranate, and cranberry can be prepared as cold beverages with natural bright colors which can be easily taken as a daily routine. Such preparations with medicinal properties without having the appearance of a typical medicine are more welcomed by patients since diabetic patients usually receive lots of conventional medicines and thus the addition of a pack of healthy biscuits or a glass of natural juice would be more pleasant than another series of capsules/tablets.

Some of the abovementioned medicinal plants have strong evidence to support their beneficial effects in CVD of T2DM; however, some others have only limited data regarding their safety and efficacy. Also, some medicinal plants have controversial data obtained in different clinical trials. One of the obvious reasons explaining these controversial results is the difference between study duration and sample size. Some biomarkers need a specific minimal time to be changed as the results of an intervention which is not considered in some trials. For instance, $\mathrm{HbAlc}$, as the gold standard of glycemic control, needs a 1.5 to 2 months of time to show the results of the intervention, whereas some clinical trials are designed for a shorter period of time, resulting in nonsignificant inter/intragroup difference which may be wrongly considered as a negative result [155].

The dosage and formulations are other important factors affecting the result of a study. Some phytochemicals such as curcuminoids in turmeric have highly lipophilic structures, causing a poor oral bioavailability; thus, bioavailabilityenhanced formulations provide higher serum concentrations of the active ingredients and consequently better clinical outcomes. Another important reason which usually remains undiscussed is the different baseline characteristics of the selected patients. Several factors including the onset of T2DM, duration of previous pharmacotherapy to control the disease, and patient adherence to the prescribed medicines, as well as genetic factors such as the race and family history of T2DM, can cause different treatment responses to the same therapeutic interventions and should be carefully considered when comparing the outcomes of different trials on the same plant. Thus, negative results obtained in patients with a long history of T2DM, usually evident from the level of $\mathrm{HbAlc}$, do not necessarily mean that the intervention cannot be effective in newly diagnosed patients [155].

The amount of main active ingredients in the herbal preparation is another factor which may affect the final results. As it has been discussed in several previous literature $[156,157]$, the amount of phytochemicals depends on several factors such as the time of harvest, storage condition, and extraction method. Lack of optimization of the production procedure results in nonuniform preparations which can affect the clinical outcome. The best way to solve this problem is to consider an optimum standardization procedure based on the major components of the plant. This can be based on a precise technique such as high-performance liquid chromatography (HPLC) or an easier but faster method such as colorimetric spectroscopic techniques. 
TABLE 2: Major possible mechanisms attributed to different phytochemical categories as protective agents in cardiovascular complications of type 2 diabetes mellitus.

\begin{tabular}{lc}
\hline Phytochemical categories & Possible mechanisms \\
\hline $\begin{array}{l}\text { Flavonoids, anthocyanins, and other } \\
\text { polyphenols }\end{array}$ & $\begin{array}{c}\text { Antioxidant, anti-inflammatory, and cytoprotective properties } \\
\text { Volatile terpenes and terpenoids } \\
\text { Nonvolatile terpenes and terpenoids } \\
\text { Fibers }\end{array}$ \\
$\begin{array}{l}\text { Sulfated compounds } \\
\text { Alkaloids }\end{array}$ & $\begin{array}{c}\text { Anti-inflammatory, antihyperglycemic, antihypertensive, and vasculoprotective properties } \\
\text { Cytoprotective effects on cardiomyocytes, antihypertensive and antihyperlipidemic effects, and } \\
\text { anticoagulant activities }\end{array}$ \\
\hline
\end{tabular}

Announcing the level of main active compounds of the herbal preparations in the clinical trials can help comparing the quality of preparations in different studies and gives a more reliable judgement regarding the obtained results.

One of the limitations of our study is that the types of interventions were diverse; thus, the results cannot be subjected to statistical analysis since they do not fulfill the essential criteria for systematic reviews and meta-analysis. However, the collected information is valuable as a comprehensive interpretation of the current clinical evidence on the management of CVD in T2DM by medicinal plants. Another limitation is that only placebo-controlled trials were included in this paper. These criteria were considered in order to include fewer studies with more homogeneity so that different interventions can be compared with each other. There are several uncontrolled studies or studies on the comparison of an herbal intervention with a positive control which can also provide valuable data and may be the subject of future review articles on the same topic.

\section{Conclusions}

Overall, several plants are capable of improving the cardiovascular complications of T2DM and can be suggested as complementary therapies or dietary interventions along with conventional medicines. Active components of the clinically effective medicinal plants can also be used as new backbones to develop semisynthetic structures with higher potency and controlled profile of adverse effects for CVD. Further preclinical studies to clarify the exact cellular and subcellular mechanisms of these natural products, as well as well-designed clinical studies, are necessary to confirm the safety and efficacy of plant-based therapies for the management of CVD in T2DM.

\section{Conflicts of Interest}

Authors declare no conflicts of interest.

\section{Authors' Contributions}

$\mathrm{RB}$ performed the search, edited the tables, and wrote the main body of the manuscript. MF and RR designed the study, prepared the table, and edited the final manuscript. MR and SN prepared the tables and edited the final manuscript.

\section{Acknowledgments}

This study was partially supported by Tehran University of Medical Sciences (TUMS), Grant no. 96-04-86-37025.

\section{References}

[1] Y. Zheng, S. H. Ley, and F. B. Hu, "Global aetiology and epidemiology of type 2 diabetes mellitus and its complications," Nature Reviews Endocrinology, vol. 14, no. 2, pp. 88-98, 2018.

[2] P. Z. Zimmet, D. J. Magliano, W. H. Herman, and J. E. Shaw, "Diabetes: a 21st century challenge," The Lancet Diabetes \& Endocrinology, vol. 2, no. 1, pp. 56-64, 2014.

[3] S. Wild, G. Roglic, A. Green, R. Sicree, and H. King, "Global prevalence of diabetes: estimates for the year 2000 and projections for 2030," Diabetes Care, vol. 27, no. 5, pp. 1047-1053, 2004.

[4] A. Norhammar, L. Mellbin, and F. Cosentino, "Diabetes: prevalence, prognosis and management of a potent cardiovascular risk factor," European Journal of Preventive Cardiology, vol. 24, no. 3, pp. 52-60, 2017.

[5] N. J. Morrish, S. L. Wang, L. K. Stevens, J. H. Fuller, and H. Keen, "Mortality and causes of death in the WHO multinational study of vascular disease in diabetes," Diabetologia, vol. 44, no. 2, pp. S14-S21, 2001.

[6] W. B. Kannel and D. L. McGee, "Diabetes and cardiovascular disease," JAMA, vol. 241, no. 19, pp. 2035-2038, 1979.

[7] D. Aune, T. Feng, S. Schlesinger, I. Janszky, T. Norat, and E. Riboli, "Diabetes mellitus, blood glucose and the risk of atrial fibrillation: a systematic review and meta-analysis of cohort studies," Journal of Diabetes and Its Complications, vol. 32, no. 5, pp. 501-511, 2018.

[8] D. Aune, S. Schlesinger, T. Norat, and E. Riboli, "Diabetes mellitus and the risk of sudden cardiac death: a systematic review and meta-analysis of prospective studies," Nutrition, Metabolism, and Cardiovascular Diseases, vol. 28, no. 6, pp. 543-556, 2018.

[9] J. R. Petrie, T. J. Guzik, and R. M. Touyz, "Diabetes, hypertension, and cardiovascular disease: clinical insights and vascular mechanisms," Canadian Journal of Cardiology, vol. 34, no. 5, pp. 575-584, 2018.

[10] M. D. Klajda, C. G. Scott, R. J. Rodeheffer, and H. H. Chen, "Diabetes mellitus is associated with increased incidence of CHF independent of hypertension and coronary artery disease: a population based study," Journal of Cardiac Failure, vol. 24, no. 8, p. S70, 2018.

[11] M. Casula, F. Mozzanica, L. Scotti et al., "Statin use and risk of new-onset diabetes: a meta-analysis of observational 
studies," Nutrition, Metabolism, and Cardiovascular Diseases, vol. 27, no. 5, pp. 396-406, 2017.

[12] M. Abdul-Ghani, R. A. DeFronzo, S. Del Prato, R. Chilton, R. Singh, and R. E. J. Ryder, "Cardiovascular disease and type 2 diabetes: has the dawn of a new era arrived?" Diabetes Care, vol. 40, no. 7, pp. 813-820, 2017.

[13] J. L. Pallisgaard, M. M. Brooks, B. R. Chaitman, D. B. Boothroyd, M. Perez, and M. A. Hlatky, "Thiazolidinediones and risk of atrial fibrillation among patients with diabetes and coronary disease," The American Journal of Medicine, vol. 131, no. 7, pp. 805-812, 2018.

[14] B. Lecka-Czernik, "Diabetes, bone and glucose-lowering agents: basic biology," Diabetologia, vol. 60, no. 7, pp. 1163-1169, 2017.

[15] A. Karimi, A. Zargaran, and A. Borhani-Haghighi, "Avicenna's description of Willis circle," International Journal of Cardiology, vol. 168, no. 3, p. 3041, 2013.

[16] M. M. Zarshenas and A. Zargaran, "A review on the Avicenna's contribution to the field of cardiology," International Journal of Cardiology, vol. 182, pp. 237-241, 2015.

[17] R. Bahramsoltani, F. Ebrahimi, M. H. Farzaei et al., "Dietary polyphenols for atherosclerosis: a comprehensive review and future perspectives," Critical Reviews in Food Science and Nutrition, vol. 59, pp. 114-132, 2017.

[18] H. Rouhi-Boroujeni, E. Heidarian, H. Rouhi-Boroujeni, F. Deris, and M. Rafieian-Kopaei, "Medicinal plants with multiple effects on cardiovascular diseases: a systematic review," Current Pharmaceutical Design, vol. 23, no. 7, pp. 999-1015, 2017.

[19] Y.-J. Xu, P. S. Tappia, N. S. Neki, and N. S. Dhalla, "Prevention of diabetes-induced cardiovascular complications upon treatment with antioxidants," Heart Failure Reviews, vol. 19, no. 1, pp. 113-121, 2014.

[20] A. R. Jadad, R. A. Moore, D. Carroll et al., "Assessing the quality of reports of randomized clinical trials: is blinding necessary?" Controlled Clinical Trials, vol. 17, no. 1, pp. 1-12, 1996.

[21] S. Ebrahimpour-Koujan, B. P. Gargari, M. Mobasseri, H. Valizadeh, and M. Asghari-Jafarabadi, "Lower glycemic indices and lipid profile among type 2 diabetes mellitus patients who received novel dose of Silybum marianum (L.) Gaertn. (silymarin) extract supplement: a triple-blinded randomized controlled clinical trial," Phytomedicine, vol. 44, pp. 39-44, 2018.

[22] S. Ebrahimpour Koujan, B. P. Gargari, M. Mobasseri, H. Valizadeh, and M. Asghari-Jafarabadi, "Effects of Silybum marianum (L.) Gaertn. (silymarin) extract supplementation on antioxidant status and hs-CRP in patients with type 2 diabetes mellitus: a randomized, triple-blind, placebo-controlled clinical trial," Phytomedicine, vol. 22, no. 2, pp. 290-296, 2015.

[23] P. Nazni, T. Poongodi Vijayakumar, P. Alagianambi, and M. Amirthaveni, "Hypoglycemic and hypolipidemic effect of Cynara scolymus among selected type 2 diabetic individuals," Pakistan Journal of Nutrition, vol. 5, no. 2, pp. 147-151, 2006.

[24] H. Fallah Huseini, S. Kianbakht, and R. Heshmat, "Cynara scolymus L. in treatment of hypercholesterolemic type 2 diabetic patients: a randomized double-blind placebo-controlled clinical trial," Journal of Medicinal Plants, vol. 1, no. 41, pp. 85-65, 2012.

[25] R. Ashraf, K. Aamir, A. R. Shaikh, and T. Ahmed, "Effects of garlic on dyslipidemia in patients with type 2 diabetes mellitus," Journal of Ayub Medical College, Abbottabad: JAMC, vol. 17, no. 3, pp. 60-64, 2005.

[26] R. Ashraf, R. A. Khan, and I. Ashraf, "Garlic (Allium sativum) supplementation with standard antidiabetic agent provides better diabetic control in type 2 diabetes patients," Pakistan Journal of Pharmaceutical Sciences, vol. 24, no. 4, pp. 565-570, 2011.

[27] M. Atkin, D. Laight, and M. H. Cummings, "The effects of garlic extract upon endothelial function, vascular inflammation, oxidative stress and insulin resistance in adults with type 2 diabetes at high cardiovascular risk. A pilot double blind randomized placebo controlled trial," Journal of Diabetes and Its Complications, vol. 30, no. 4, pp. 723-727, 2016.

[28] S. Mahluji, V. E. Attari, M. Mobasseri, L. Payahoo, A. Ostadrahimi, and S. E. Golzari, "Effects of ginger (Zingiber officinale) on plasma glucose level, $\mathrm{HbAlc}$ and insulin sensitivity in type 2 diabetic patients," International Journal of Food Sciences \& Nutrition, vol. 64, no. 6, pp. 682-686, 2013.

[29] T. Arablou, N. Aryaeian, M. Valizadeh, F. Sharifi, A. Hosseini, and M. Djalali, "The effect of ginger consumption on glycemic status, lipid profile and some inflammatory markers in patients with type 2 diabetes mellitus," International Journal of Food Sciences \& Nutrition, vol. 65, no. 4, pp. 515-520, 2014.

[30] F. Shidfar, A. Rajab, T. Rahideh, N. Khandouzi, S. Hosseini, and S. Shidfar, "The effect of ginger (Zingiber officinale) on glycemic markers in patients with type 2 diabetes," Journal of Complementary \& Integrative Medicine, vol. 12, no. 2, pp. 165-170, 2015.

[31] S. Hosseini, M. Alipour, M. Zakerkish, and M. H. Haghighizadeh, "Effects of standardized extract of ginseng (G115) on biomarkers of systemic low-grade inflammation in patients with type 2 diabetes: a double-blind clinical trial," Iranian Journal of Endocrinology and Metabolism, vol. 16, no. 3, pp. 175-182, 2014.

[32] N. Namazi, A. T. Esfanjani, J. Heshmati, and A. Bahrami, "The effect of hydro alcoholic Nettle (Urtica dioica) extracts on insulin sensitivity and some inflammatory indicators in patients with type 2 diabetes: a randomized double-blind control trial," Pakistan Journal of Biological Sciences, vol. 14, no. 15, pp. 775-779, 2011.

[33] A. A. Behzadi, H. Kalalian-Moghaddam, and A. H. Ahmadi, "Effects of Urtica dioica supplementation on blood lipids, hepatic enzymes and nitric oxide levels in type 2 diabetic patients: a double blind, randomized clinical trial," Avicenna Journal of Phytomedicine, vol. 6, no. 6, p. 686, 2016.

[34] G. Sohrab, S. Ebrahimof, G. Sotoudeh et al., "Effects of pomegranate juice consumption on oxidative stress in patients with type 2 diabetes: a single-blind, randomized clinical trial," International Journal of Food Sciences \& Nutrition, vol. 68, no. 2, pp. 249-255, 2017.

[35] F. Shidfar, I. Heydari, S. J. Hajimiresmaiel, S. Hosseini, S. Shidfar, and F. Amiri, "The effects of cranberry juice on serum glucose, apoB, apoA-I, Lp(a), and paraoxonase-1 activity in type 2 diabetic male patients," Journal of Research in Medical Sciences: The Official Journal of Isfahan University of Medical Sciences, vol. 17, no. 4, pp. 355-360, 2012.

[36] P. Kar, D. Laight, H. K. Rooprai, K. M. Shaw, and M. Cummings, "Effects of grape seed extract in type 2 diabetic subjects at high cardiovascular risk: a double blind randomized placebo controlled trial examining metabolic markers, vascular tone, inflammation, oxidative stress and 
insulin sensitivity," Diabetic Medicine, vol. 26, no. 5, pp. 526-531, 2009.

[37] I. Mucalo, E. Jovanovski, D. Rahelić, V. Božikov, Ž. Romić, and V. Vuksan, "Effect of American ginseng (Panax quinquefolius L.) on arterial stiffness in subjects with type-2 diabetes and concomitant hypertension," Journal of Ethnopharmacology, vol. 150, no. 1, pp. 148-153, 2013.

[38] F. Lazavi, P. Mirmiran, G. Sohrab, O. Nikpayam, P. Angoorani, and M. Hedayati, "The barberry juice effects on metabolic factors and oxidative stress in patients with type 2 diabetes: a randomized clinical trial," Complementary Therapies in Clinical Practice, vol. 31, pp. 170-174, 2018.

[39] F. Shidfar, S. S. Ebrahimi, S. Hosseini, I. Heydari, S. Shidfar, and G. Hajhassani, "The effects of Berberis vulgaris fruit extract on serum lipoproteins, apoB, apoA-I, homocysteine, glycemic control and total antioxidant capacity in type 2 diabetic patients," Iranian Journal of Pharmaceutical Research, vol. 11, no. 2, pp. 643-652, 2012.

[40] R. K. Sharma, B. Sharma, M. Jindal et al., "Evaluation of hypolipidemic effect of stem part of Berberis aristata in type 2 diabetes mellitus patients as add on therapy," National Journal of Physiology, Pharmacy and Pharmacology, vol. 7, no. 11, pp. 1A-11A, 2017.

[41] S. Qian, S. Wang, P. Fan, D. Huo, L. Dai, and Q. Qian, "Effect of Salvia miltiorrhiza hydrophilic extract on the endothelial biomarkers in diabetic patients with chronic artery disease," Phytotherapy Research, vol. 26, no. 10, pp. 1575-1578, 2012.

[42] X.-s. Li, W.-y. Zheng, S.-x. Lou, X.-w. Lu, and S.-h. Ye, "Effect of Ginkgo leaf extract on vascular endothelial function in patients with early stage diabetic nephropathy," Chinese Journal of Integrative Medicine, vol. 15, no. 1, pp. 26-29, 2009.

[43] V. Dall'Alba, F. M. Silva, J. P. Antonio et al., "Improvement of the metabolic syndrome profile by soluble fibre-guar gumin patients with type 2 diabetes: a randomised clinical trial," British Journal of Nutrition, vol. 110, no. 9, pp. 1601-1610, 2013.

[44] X. Ma, J. Gu, Z. Zhang et al., "Effects of Avena nuda L. on metabolic control and cardiovascular disease risk among Chinese patients with diabetes and meeting metabolic syndrome criteria: secondary analysis of a randomized clinical trial," European Journal of Clinical Nutrition, vol. 67, no. 12, pp. 1291-1297, 2013.

[45] M. Taghavi, R. Farid Hoseini, H. Rafat Panah, M. Sharifian Razavi, and R. Watson, "Effect of feijoa supplementation in patients with type 2 diabetes," Iranian Journal of Endocrinology and Metabolism, vol. 14, no. 3, pp. 222-225, 2012.

[46] I. A. Sobenin, L. V. Nedosugova, L. V. Filatova, M. I. Balabolkin, T. V. Gorchakova, and A. N. Orekhov, "Metabolic effects of time-released garlic powder tablets in type 2 diabetes mellitus: the results of double-blinded placebo-controlled study," Acta Diabetologica, vol. 45, no. 1, pp. 1-6, 2008.

[47] H. F. Huseini, S. Kianbakht, R. Hajiaghaee, and F. H. Dabaghian, "Anti-hyperglycemic and anti-hypercholesterolemic effects of Aloe vera leaf gel in hyperlipidemic type 2 diabetic patients: a randomized double-blind placebocontrolled clinical trial," Planta Medica, vol. 78, no. 4, pp. 311-316, 2012.

[48] A. Zarrintan, M. Mobasseri, A. Zarrintan, and A. Ostadrahimi, "Effects of aloe vera supplements on blood glucose level and lipid profile markers in type 2 diabetic patients-a randomized clinical trial," Pharmaceutical Sciences, vol. 21, no. 2, pp. 65-71, 2015.
[49] A. Azadmehr, A. Ziaee, L. Ghanei et al., "A randomized clinical trial study: anti-oxidant, anti-hyperglycemic and anti-hyperlipidemic effects of olibanum gum in type 2 diabetic patients," Iranian Journal of Pharmaceutical Research, vol. 13, no. 3, pp. 1003-1009, 2014.

[50] D. J. A. Jenkins, C. W. C. Kendall, V. Vuksan et al., "Effect of lowering the glycemic load with canola oil on glycemic control and cardiovascular risk factors: a randomized controlled trial," Diabetes Care, vol. 37, no. 7, pp. 1806-1814, 2014.

[51] Z. Bahadoran, P. Mirmiran, F. Hosseinpanah, A. Rajab, G. Asghari, and F. Azizi, "Broccoli sprouts powder could improve serum triglyceride and oxidized LDL/LDL-cholesterol ratio in type 2 diabetic patients: a randomized doubleblind placebo-controlled clinical trial," Diabetes Research and Clinical Practice, vol. 96, no. 3, pp. 348-354, 2012.

[52] J. Somanah, O. I. Aruoma, T. K. Gunness et al., "Effects of a short term supplementation of a fermented papaya preparation on biomarkers of diabetes mellitus in a randomized Mauritian population," Preventive Medicine, vol. 54, pp. S90-S97, 2012.

[53] A. Khan, M. Safdar, M. M. Ali Khan, K. N. Khattak, and R. A. Anderson, "Cinnamon improves glucose and lipids of people with type 2 diabetes," Diabetes Care, vol. 26, no. 12, pp. 3215-3218, 2003.

[54] J. Wainstein, N. Stern, S. Heller, and M. Boaz, "Dietary cinnamon supplementation and changes in systolic blood pressure in subjects with type 2 diabetes," Journal of $\mathrm{Me}$ dicinal Food, vol. 14, no. 12, pp. 1505-1510, 2011.

[55] B. Talaei, A. Amouzegar, S. Sahranavard, M. Hedayati, P. Mirmiran, and F. Azizi, "Effects of cinnamon consumption on glycemic indicators, advanced glycation end products, and antioxidant status in type 2 diabetic patients," Nutrients, vol. 9, 2017.

[56] E. Dalli, E. Colomer, M. C. Tormos et al., "Crataegus laevigata decreases neutrophil elastase and has hypolipidemic effect: a randomized, double-blind, placebo-controlled trial," Phytomedicine: International Journal of Phytotherapy and Phytopharmacology, vol. 18, no. 8-9, pp. 769-775, 2011.

[57] A. F. Walker, G. Marakis, E. Simpson et al., "Hypotensive effects of hawthorn for patients with diabetes taking prescription drugs: a randomised controlled trial," British Journal of General Practice, vol. 56, no. 527, pp. 437-443, 2006.

[58] A. Moravej Aleali, R. Amani, H. Shahbazian, F. Namjooyan, S. M. Latifi, and B. Cheraghian, "The effect of hydroalcoholic saffron (Crocus sativus L.) extract on fasting plasma glucose, $\mathrm{HbAlc}$, lipid profile, liver, and renal function tests in patients with type 2 diabetes mellitus: a randomized double-blind clinical trial," Phytotherapy Research, vol. 33, no. 6, pp. 1648-1657, 2019.

[59] A. Bayat, F. Azizi-Soleiman, M. Heidari-Beni et al., "Effect of Cucurbita ficifolia and probiotic yogurt consumption on blood glucose, lipid profile, and inflammatory marker in type 2 diabetes," International Journal of Preventive Medicine, vol. 7, p. 30, 2016.

[60] S. Chuengsamarn, S. Rattanamongkolgul, B. Phonrat, R. Tungtrongchitr, and S. Jirawatnotai, "Reduction of atherogenic risk in patients with type 2 diabetes by curcuminoid extract: a randomized controlled trial," The Journal of $\mathrm{Nu}$ tritional Biochemistry, vol. 25, no. 2, pp. 144-150, 2014.

[61] A. Srinivasan, S. Selvarajan, S. Kamalanathan, T. Kadhiravan, N. C. Prasanna Lakshmi, and S. Adithan, "Effect of Curcuma longa on vascular function in native Tamilians with type 2 
diabetes mellitus: a randomized, double-blind, parallel arm, placebo-controlled trial," Phytotherapy Research, vol. 33, no. 7, pp. 1898-1911, 2019.

[62] Z. Adab, S. Eghtesadi, M. R. Vafa et al., "Effect of turmeric on glycemic status, lipid profile, hs-CRP, and total antioxidant capacity in hyperlipidemic type 2 diabetes mellitus patients," Phytotherapy Research, vol. 33, no. 4, pp. 1173-1181, 2019.

[63] D. Kuate, B. C. Etoundi, J. L. Ngondi, and J. E. Oben, "Effects of Dichrostachys glomerata spice on cardiovascular diseases risk factors in normoglycemic and type 2 diabetic obese volunteers," Food Research International, vol. 44, no. 5, pp. 1197-1202, 2011.

[64] S. Moazen, R. Amani, A. Homayouni Rad, H. Shahbazian, K. Ahmadi, and M. Taha Jalali, "Effects of freeze-dried strawberry supplementation on metabolic biomarkers of atherosclerosis in subjects with type 2 diabetes: a randomized double-blind controlled trial," Annals of Nutrition and Metabolism, vol. 63, no. 3, pp. 256-264, 2013.

[65] M. Kusunoki, D. Sato, K. Tsutsumi, H. Tsutsui, T. Nakamura, and Y. Oshida, "Black soybean extract improves lipid profiles in fenofibrate-treated type 2 diabetics with postprandial hyperlipidemia," Journal of Medicinal Food, vol. 18, no. 6, pp. 615-618, 2015.

[66] A. Sedaghat, H. Shahbazian, A. Rezazadeh et al., "The effect of soy nut on serum total antioxidant, endothelial function and cardiovascular risk factors in patients with type 2 diabetes," Diabetes \& Metabolic Syndrome: Clinical Research Reviews, vol. 13, no. 2, pp. 1387-1391, 2019.

[67] T. Bandara, B. Rokeya, S. Khan et al., "Effects of Gymnema lactiferum leaves on glycemic and lipidemic status in type 2 diabetes subjects," Bangladesh Journal of Pharmacology, vol. 4, pp. 92-95, 2009.

[68] Y. Ma, V. Y. Njike, J. Millet et al., "Effects of walnut consumption on endothelial function in type 2 diabetic subjects: a randomized controlled crossover trial," Diabetes Care, vol. 33, no. 2, pp. 227-232, 2010.

[69] S. Hosseini, L. Jamshidi, S. Mehrzadi et al., "Effects of Juglans regia L. leaf extract on hyperglycemia and lipid profiles in type two diabetic patients: a randomized double-blind, placebo-controlled clinical trial," Journal of Ethnopharmacology, vol. 152, no. 3, pp. 451-456, 2014.

[70] K. Rabiei, M. A. Ebrahimzadeh, M. Saeedi, A. Bahar, O. Akha, and Z. Kashi, "Effects of a hydroalcoholic extract of Juglans regia (walnut) leaves on blood glucose and major cardiovascular risk factors in type 2 diabetic patients: a double-blind, placebo-controlled clinical trial," BMC Complementary and Alternative Medicine, vol. 18, no. 1, p. 206, 2018.

[71] A. Aljamal, "Effects of bay leaves on the patients with diabetes mellitus," Research Journal of Medicinal Plant, vol. 5, no. 4, pp. 471-476, 2011.

[72] G. Thakur, A. Mitra, K. Pal, and D. Rousseau, "Effect of flaxseed gum on reduction of blood glucose and cholesterol in type 2 diabetic patients," International Journal of Food Sciences \& Nutrition, vol. 60, no. 6, pp. 126-136, 2009.

[73] J. E. Upritchard, W. H. Sutherland, and J. I. Mann, "Effect of supplementation with tomato juice, vitamin E, and vitamin C on LDL oxidation and products of inflammatory activity in type 2 diabetes," Diabetes Care, vol. 23, no. 6, pp. 733-738, 2000.

[74] A. Asadi, F. Shidfar, M. Safari et al., "Safety and efficacy of Melissa officinalis (lemon balm) on ApoA-I, Apo B, lipid ratio and ICAM-1 in type 2 diabetes patients: a randomized, double-blinded clinical trial," Complementary Therapies in Medicine, vol. 40, pp. 83-88, 2018.

[75] D. M. Riche, K. D. Riche, H. E. East, E. K. Barrett, and W. L. May, "Impact of mulberry leaf extract on type 2 diabetes (Mul-DM): a randomized, placebo-controlled pilot study," Complementary Therapies in Medicine, vol. 32, pp. 105-108, 2017.

[76] V. Vuksan, Z. Z. Xu, E. Jovanovski et al., "Efficacy and safety of American ginseng (Panax quinquefolius L.) extract on glycemic control and cardiovascular risk factors in individuals with type 2 diabetes: a double-blind, randomized, cross-over clinical trial," European Journal of Nutrition, vol. 58, no. 3, pp. 1237-1245, 2018.

[77] I. N. Raju, K. K. Reddy, C. K. Kumari et al., "Efficacy of purple passion fruit peel extract in lowering cardiovascular risk factors in type 2 diabetic subjects," Journal of EvidenceBased Complementary \& Alternative Medicine, vol. 18, no. 3, pp. 183-190, 2013.

[78] P. Usharani, N. Fatima, and N. Muralidhar, "Effects of Phyllanthus emblica extract on endothelial dysfunction and biomarkers of oxidative stress in patients with type 2 diabetes mellitus: a randomized, double-blind, controlled study," Diabetes, Metabolic Syndrome and Obesity: Targets and Therapy, vol. 6, pp. 275-284, 2013.

[79] S. Zibadi, P. J. Rohdewald, D. Park, and R. R. Watson, "Reduction of cardiovascular risk factors in subjects with type 2 diabetes by pycnogenol supplementation," Nutrition Research, vol. 28, no. 5, pp. 315-320, 2008.

[80] S. Ziai, B. Larijani, H. Fakhrzadeh et al., "Study of Psyllium (Plantago ovata L.) effects on diabetes and lipidemia in the Iranian type II diabetic patients," Journal of Medicinal Plants, vol. 4, no. 12, pp. 33-42, 2004.

[81] A. Esmaillzadeh, E. Zakizadeh, E. Faghihimani, M. Gohari, and S. Jazayeri, "The effect of purslane seeds on glycemic status and lipid profiles of persons with type 2 diabetes: a randomized controlled cross-over clinical trial," Journal of Research in Medical Sciences: The Official Journal of Isfahan University of Medical Sciences, vol. 20, no. 1, pp. 47-53, 2015.

[82] J. Wainstein, Z. Landau, Y. B. Dayan et al., "Purslane extract and glucose homeostasis in adults with type 2 diabetes: a double-blind, placebo-controlled clinical trial of efficacy and safety," Journal of Medicinal Food, vol. 19, no. 2, pp. 133-140, 2016.

[83] J.-F. Liu, Y.-H. Liu, C.-M. Chen, W.-H. Chang, and C.-Y. O. Chen, "The effect of almonds on inflammation and oxidative stress in Chinese patients with type 2 diabetes mellitus: a randomized crossover controlled feeding trial," European Journal of Nutrition, vol. 52, no. 3, pp. 927-935, 2013.

[84] S.-C. Li, Y.-H. Liu, J.-F. Liu, W.-H. Chang, C.-M. Chen, and C.-Y. O. Chen, "Almond consumption improved glycemic control and lipid profiles in patients with type 2 diabetes mellitus," Metabolism, vol. 60, no. 4, pp. 474-479, 2011.

[85] H. Fallah Huseini, R. Heshmat, F. Mohseni et al., "The efficacy of Rheum ribes L. stalk extract on lipid profile in hypercholesterolemic type II diabetic patients: a randomized, double-blind, placebo-controlled, clinical trial," Journal of Medicinal Plants, vol. 3, no. 27, pp. 92-97, 2008.

[86] S. Kianbakht and F. H. Dabaghian, "Improved glycemic control and lipid profile in hyperlipidemic type 2 diabetic patients consuming Salvia officinalis L. leaf extract: a randomized placebo. Controlled clinical trial," Complementary Therapies in Medicine, vol. 21, no. 5, pp. 441-446, 2013. 
[87] S. Vosough-Ghanbari, R. Rahimi, S. Kharabaf et al., "Effects of Satureja khuzestanica on serum glucose, lipids and markers of oxidative stress in patients with type 2 diabetes mellitus: a double-blind randomized controlled trial," Evidence-Based Complementary and Alternative Medicine, vol. 7, no. 4, pp. 465-470, 2010.

[88] P. Mirmiran, Z. Bahadoran, M. Golzarand, A. Rajab, and F. Azizi, "Ardeh (Sesamum indicum) could improve serum triglycerides and atherogenic lipid parameters in type 2 diabetic patients: a randomized clinical trial," Archives of Iranian Medicine, vol. 16, no. 11, pp. 651-656, 2013.

[89] H. F. Huseini, B. Larijani, H. Fakhrzaeh et al., "The clinical trial of Silybum marianum seed extract (silymarin) on type II diabetic patients with hyperlipidemia," Journal of Diabetes and Metabolic Disorders, vol. 3, p. 78, 2004.

[90] A. Taleb, F. Ghanadi, S. Changizi Ashtiani et al., "The effect of aqueous extract thymus Kotschyanus boiss. et hohen on glycemic control and dyslipidemia associated with type II diabetes: a randomized controlled trial," Iranian Journal of Endocrinology and Metabolism, vol. 19, no. 4, pp. 234-243, 2017.

[91] I. T. Lee, Y. C. Chan, C. W. Lin, W. J. Lee, and W. H.-H. Sheu, "Effect of cranberry extracts on lipid profiles in subjects with type 2 diabetes," Diabetic Medicine, vol. 25, no. 12, pp. 1473-1477, 2008.

[92] S. Abedini, B. P. Gargari, H. Babayi, A. A. Asgarzadeh, and P. Pourabdollahi, "Effect of supplementation with grape seed extract (Vitis vinifera) on serum lipid profiles in patient with type 2 diabetes," Iranian Journal of Endocrinology and Metabolism, vol. 15, no. 1, pp. 59-66, 2013.

[93] S. P. Dongerkery, P. R. Schroeder, and M. E. Shomali, "Insulin and its cardiovascular effects: what is the current evidence?" Current Diabetes Reports, vol. 17, no. 12, p. 120, 2017.

[94] B. Luo, Y. Soesanto, and D. A. McClain, "Protein modification by O-linked GlcNAc reduces angiogenesis by inhibiting Akt activity in endothelial cells," Arteriosclerosis, Thrombosis, and Vascular Biology, vol. 28, no. 4, pp. 651-657, 2008.

[95] N. Katakami, "Mechanism of development of atherosclerosis and cardiovascular disease in diabetes mellitus," Journal of Atherosclerosis and Thrombosis, vol. 25, no. 1, pp. 27-39, 2018.

[96] M. Bahmani, H. Shirzad, S. Rafieian, and M. Rafieian-Kopaei, "Silybum marianum: beyond hepatoprotection," Journal of Evidence-Based Complementary \& Alternative Medicine, vol. 20, no. 4, pp. 292-301, 2015.

[97] A. Taleb, K. A. Ahmad, A. U. Ihsan et al., "Antioxidant effects and mechanism of silymarin in oxidative stress induced cardiovascular diseases," Biomedicine \& Pharmacotherapy, vol. 102, pp. 689-698, 2018.

[98] M.-R. Taskinen and J. Borén, "New insights into the pathophysiology of dyslipidemia in type 2 diabetes," Atherosclerosis, vol. 239, no. 2, pp. 483-495, 2015.

[99] M. P. Hermans and P. Valensi, "Elevated triglycerides and low high-density lipoprotein cholesterol level as marker of very high risk in type 2 diabetes," Current Opinion in Endocrinology Diabetes and Obesity, vol. 25, no. 2, pp. 118-129, 2018.

[100] T. Nakano, K. Nakajima, M. Niimi et al., "Detection of apolipoproteins B-48 and B-100 carrying particles in lipoprotein fractions extracted from human aortic atherosclerotic plaques in sudden cardiac death cases," Clinica chimica acta; international journal of clinical chemistry, vol. 390, no. 1-2, pp. 38-43, 2008.

[101] K. K. Ray, S. R. K. Seshasai, S. Wijesuriya et al., "Effect of intensive control of glucose on cardiovascular outcomes and death in patients with diabetes mellitus: a meta-analysis of randomised controlled trials," The Lancet, vol. 373, no. 9677, pp. 1765-1772, 2009.

[102] A. Abbasi, E. Corpeleijn, R. T. Gansevoort et al., "Role of HDL cholesterol and estimates of HDL particle composition in future development of type 2 diabetes in the general population: the PREVEND study," Journal of Clinical Endocrinology \& Metabolism, vol. 98, no. 8, pp. E1352-E1359, 2013.

[103] J. D. Schofield, Y. Liu, P. Rao-Balakrishna, R. A. Malik, and H. Soran, "Diabetes dyslipidemia," Diabetes Therapy, vol. 7, no. 2, pp. 203-219, 2016.

[104] B. Vergès, "Pathophysiology of diabetic dyslipidaemia: where are we?” Diabetologia, vol. 58, no. 5, pp. 886-899, 2015.

[105] X. Li, J. Ni, Y. Tang et al., "Allicin inhibits mouse colorectal tumorigenesis through suppressing the activation of STAT3 signaling pathway," Natural Product Research, vol. 33, pp. 2722-2725, 2018.

[106] X. L. Louis, R. Murphy, S. J. Thandapilly, L. Yu, and T. Netticadan, "Garlic extracts prevent oxidative stress, hypertrophy and apoptosis in cardiomyocytes: a role for nitric oxide and hydrogen sulfide," BMC Complementary and Alternative Medicine, vol. 12, no. 1, p. 140, 2012.

[107] R. Shouk, A. Abdou, K. Shetty, D. Sarkar, and A. H. Eid, "Mechanisms underlying the antihypertensive effects of garlic bioactives," Nutrition Research, vol. 34, no. 2, pp. 106-115, 2014.

[108] R. Kumar, S. Chhatwal, S. Arora et al., "Antihyperglycemic, antihyperlipidemic, anti-inflammatory and adenosine deaminase-lowering effects of garlic in patients with type 2 diabetes mellitus with obesity," Diabetes, Metabolic Syndrome and Obesity: Targets and Therapy, vol. 6, pp. 49-56, 2013.

[109] F. Q. Alali, T. El-Elimat, L. Khalid, R. Hudaib, T. S. AlShehabi, and A. H. Eid, "Garlic for cardiovascular disease: prevention or treatment?" Current Pharmaceutical Design, vol. 23, no. 7, pp. 1028-1041, 2017.

[110] R. Nicoll and M. Y. Henein, "Ginger (Zingiber officinale Roscoe): a hot remedy for cardiovascular disease?" International Journal of Cardiology, vol. 131, no. 3, pp. 408-409, 2009.

[111] A. D. Pradhan, J. E. Manson, N. Rifai, J. E. Buring, and P. M. Ridker, "C-reactive protein, interleukin 6, and risk of developing type 2 diabetes mellitus," JAMA, vol. 286, no. 3, pp. 327-334, 2001.

[112] M. Y. Donath and S. E. Shoelson, "Type 2 diabetes as an inflammatory disease," Nature Reviews Immunology, vol. 11, no. 2, pp. 98-107, 2011.

[113] M. C. Calle and M. L. Fernandez, "Inflammation and type 2 diabetes," Diabetes \& Metabolism, vol. 38, no. 3, pp. 183-191, 2012.

[114] P. M. Ridker, E. Danielson, F. A. H. Fonseca et al., "Rosuvastatin to prevent vascular events in men and women with elevated C-reactive protein," New England Journal of Medicine, vol. 359, no. 21, pp. 2195-2207, 2008.

[115] D. Qu, J. Liu, C. W. Lau, and Y. Huang, "IL-6 in diabetes and cardiovascular complications," British Journal of Pharmacology, vol. 171, no. 15, pp. 3595-3603, 2014.

[116] J. Lee, S. Lee, H. Zhang, M. A. Hill, C. Zhang, and Y. Park, "Interaction of IL- 6 and TNF- $\alpha$ contributes to endothelial 
dysfunction in type 2 diabetic mouse hearts," PLoS One, vol. 12, no. 11, Article ID e0187189, 2017.

[117] E. Saba, D. Jeong, M. Irfan et al., "Anti-inflammatory activity of Rg3-enriched Korean red ginseng extract in murine model of sepsis," Evidence-Based Complementary and Alternative Medicine, vol. 2018, Article ID 6874692, 11 pages, 2018.

[118] D. Jeong, M. Irfan, S.-D. Kim et al., "Ginsenoside Rg3enriched red ginseng extract inhibits platelet activation and in vivo thrombus formation," Journal of Ginseng Research, vol. 41, no. 4, pp. 548-555, 2017.

[119] M. El Haouari and J. A. Rosado, "Phytochemical, anti-diabetic and cardiovascular properties of Urtica dioica L. (Urticaceae): a review," Mini Reviews in Medicinal Chemistry, vol. 19, no. 1, pp. 63-71, 2019.

[120] R. Rahimi, S. Nikfar, B. Larijani, and M. Abdollahi, "A review on the role of antioxidants in the management of diabetes and its complications," Biomedicine \& Pharmacotherapy, vol. 59, no. 7, pp. 365-373, 2005.

[121] R. Robson, A. R. Kundur, and I. Singh, "Oxidative stress biomarkers in type 2 diabetes mellitus for assessment of cardiovascular disease risk," Diabetes \& Metabolic Syndrome: Clinical Research Reviews, vol. 12, no. 3, pp. 455-462, 2018.

[122] F. Giacco and M. Brownlee, "Oxidative stress and diabetic complications," Circulation Research, vol. 107, no. 9, pp. 1058-1070, 2010.

[123] S. Tsuzura, Y. Ikeda, T. Suehiro et al., "Correlation of plasma oxidized low-density lipoprotein levels to vascular complications and human serum paraoxonase in patients with type 2 diabetes," Metabolism, vol. 53, no. 3, pp. 297-302, 2004.

[124] S. K. Patra, K. Singh, and R. Singh, "Paraoxonase 1: a better atherosclerotic risk predictor than HDL in type 2 diabetes mellitus," Diabetes \& Metabolic Syndrome: Clinical Research Reviews, vol. 7, no. 2, pp. 108-111, 2013.

[125] F. K. Lutchmansingh, J. W. Hsu, F. I. Bennett et al., "Glutathione metabolism in type 2 diabetes and its relationship with microvascular complications and glycemia," PLoS One, vol. 13, no. 6, Article ID e0198626, 2018.

[126] A. Zarfeshany, S. Asgary, and S. H. Javanmard, "Potent health effects of pomegranate," Advanced Biomedical Research, vol. 3, p. 100, 2014.

[127] R. Naseri, F. Farzaei, P. Haratipour et al., "Anthocyanins in the management of metabolic syndrome: a pharmacological and biopharmaceutical review," Frontiers in Pharmacology, vol. 9, p. 1310, 2018.

[128] A. I. Adler, I. M. Stratton, H. A. Neil et al., "Association of systolic blood pressure with macrovascular and microvascular complications of type 2 diabetes (UKPDS 36): prospective observational study," $B M J$, vol. 321, no. 7258, pp. 412-419, 2000.

[129] L. Hansson, A. Zanchetti, S. G. Carruthers et al., "Effects of intensive blood-pressure lowering and low-dose aspirin in patients with hypertension: principal results of the hypertension optimal treatment (HOT) randomised trial," The Lancet, vol. 351, no. 9118, pp. 1755-1762, 1998.

[130] G. Lastra, S. Syed, L. R. Kurukulasuriya, C. Manrique, and J. R. Sowers, "Type 2 diabetes mellitus and hypertension," Endocrinology and Metabolism Clinics of North America, vol. 43, no. 1, pp. 103-122, 2014.

[131] L. Yvan-Charvet and A. Quignard-Boulangé, "Role of adipose tissue renin-angiotensin system in metabolic and inflammatory diseases associated with obesity," Kidney International, vol. 79, no. 2, pp. 162-168, 2011.

[132] S. Sen, S. Chen, B. Feng, Y. Wu, E. Lui, and S. Chakrabarti, "American ginseng (Panax quinquefolius) prevents glucose- induced oxidative stress and associated endothelial abnormalities," Phytomedicine, vol. 18, no. 13, pp. 1110-1117, 2011.

[133] Q. Wu, W. Wang, S. Li et al., "American ginseng inhibits vascular smooth muscle cell proliferation via suppressing Jak/Stat pathway," Journal of Ethnopharmacology, vol. 144, no. 3, pp. 782-785, 2012.

[134] Y.-G. Ma, L. Liang, Y.-B. Zhang et al., "Berberine reduced blood pressure and improved vasodilation in diabetic rats," Journal of Molecular Endocrinology, vol. 59, no. 3, pp. 191204, 2017.

[135] Y. Wang and Y. Ding, "Berberine protects vascular endothelial cells in hypertensive rats," International Journal of Clinical and Experimental Medicine, vol. 8, no. 9, pp. 14896-14905, 2015.

[136] H. Kulkarni, M. Mamtani, J. Peralta et al., "Soluble forms of intercellular and vascular cell adhesion molecules independently predict progression to type 2 diabetes in Mexican American families," PLoS One, vol. 11, no. 3, Article ID e0151177, 2016.

[137] J. B. Meigs, F. B. Hu, N. Rifai, and J. E. Manson, "Biomarkers of endothelial dysfunction and risk of type 2 diabetes mellitus," JAMA, vol. 291, no. 16, pp. 1978-1986, 2004.

[138] C. P. Domingueti, L. M. S. A. Dusse, M. d. G. Carvalho, L. P. de Sousa, K. B. Gomes, and A. P. Fernandes, "Diabetes mellitus: the linkage between oxidative stress, inflammation, hypercoagulability and vascular complications," Journal of Diabetes and Its Complications, vol. 30, no. 4, pp. 738-745, 2016.

[139] U. Campia, M. Tesauro, N. Di Daniele, and C. Cardillo, "The vascular endothelin system in obesity and type 2 diabetes: pathophysiology and therapeutic implications," Life Sciences, vol. 118, no. 2, pp. 149-155, 2014.

[140] Z.-m. Li, S.-w. Xu, and P.-q. Liu, "Salvia miltiorrhiza Burge (Danshen): a golden herbal medicine in cardiovascular therapeutics," Acta Pharmacologica Sinica, vol. 39, no. 5, pp. 802-824, 2018.

[141] H. C. Ou, Y. L. Hsieh, N. C. Yang et al., "Ginkgo biloba extract attenuates oxLDL-induced endothelial dysfunction via an AMPK-dependent mechanism," Journal of Applied Physiology, vol. 114, no. 2, pp. 274-285, 2013.

[142] Z. Xing, J. Pei, J. Huang, X. Peng, P. Chen, and X. Hu, "Relationship of obesity to adverse events among patients with mean 10-year history of type 2 diabetes mellitus: results of the ACCORD study," Journal of the American Heart Association, vol. 7, no. 22, Article ID e010512, 2018.

[143] S. E. Kahn, R. L. Hull, and K. M. Utzschneider, "Mechanisms linking obesity to insulin resistance and type 2 diabetes," Nature, vol. 444, no. 7121, pp. 840-846, 2006.

[144] X. Wen, C. Qian, Y. Zhang et al., "Key pathway and gene alterations in the gastric mucosa associated with obesity and obesity-related diabetes," Journal of Cellular Biochemistry, vol. 120, no. 4, pp. 6763-6771, 2018.

[145] X. Hou, J. Lu, J. Weng et al., "Impact of waist circumference and body mass index on risk of cardiometabolic disorder and cardiovascular disease in Chinese adults: a national diabetes and metabolic disorders survey," PLoS One, vol. 8, no. 3, Article ID e57319, 2013.

[146] R. Siren, J. G. Eriksson, and H. Vanhanen, "Waist circumference a good indicator of future risk for type 2 diabetes and cardiovascular disease," BMC Public Health, vol. 12, no. 1, p. 631, 2012.

[147] C. Koliaki, S. Liatis, and A. Kokkinos, "Obesity and cardiovascular disease: revisiting an old relationship," Metabolism, vol. 92, pp. 98-107, 2019. 
[148] D. Mudgil, S. Barak, and B. S. Khatkar, "Guar gum: processing, properties and food applications-a review," Journal of Food Science \& Technology, vol. 51, no. 3, pp. 409-418, 2014.

[149] R. Bahramsoltani, R. Rahimi, and M. H. Farzaei, "Pharmacokinetic interactions of curcuminoids with conventional drugs: a review," Journal of Ethnopharmacology, vol. 209, pp. 1-12, 2017.

[150] S. Soleymani, R. Bahramsoltani, R. Rahimi, and M. Abdollahi, "Clinical risks of St. John's Wort (Hypericum perforatum) co-administration," Expert Opinion on Drug Metabolism and Toxicology, vol. 13, no. 10, pp. 1047-1062, 2017.

[151] A. A. Izzo, G. Di Carlo, F. Borrelli, and E. Ernst, "Cardiovascular pharmacotherapy and herbal medicines: the risk of drug interaction," International Journal of Cardiology, vol. 98, no. 1, pp. 1-14, 2005.

[152] A. A. Izzo, "Interactions between herbs and conventional drugs: overview of the clinical data," Medical Principles and Practice, vol. 21, no. 5, pp. 404-428, 2012.

[153] F. Maione, C. Cicala, G. Musciacco et al., "Phenols, alkaloids and terpenes from medicinal plants with antihypertensive and vasorelaxant activities. A review of natural products as leads to potential therapeutic agents," Natural Product Communications, vol. 8, no. 4, pp. 539-544, 2013.

[154] I. A. Menezes, C. M. Barreto, A. R. Antoniolli, M. R. Santos, and D. P. de Sousa, "Hypotensive activity of terpenes found in essential oils," Zeitschrift fur Naturforschung. C, Journal of Biosciences, vol. 65, no. 9-10, pp. 562-566, 2010.

[155] E. Rezaeiamiri, R. Bahramsoltani, and R. Rahimi, "Plantderived natural agents as dietary supplements for the regulation of glycosylated hemoglobin: a review of clinical trials," Clinical Nutrition, vol. 39, no. 2, pp. 331-342, 2019.

[156] J. Rohloff, S. Dragland, R. Mordal, and T.-H. Iversen, "Effect of harvest time and drying method on biomass production, essential oil yield, and quality of peppermint (Mentha piperita L.)," Journal of Agricultural and Food Chemistry, vol. 53, no. 10, pp. 4143-4148, 2005.

[157] S. Tavarini and L. G. Angelini, "Stevia rebaudiana Bertoni as a source of bioactive compounds: the effect of harvest time, experimental site and crop age on steviol glycoside content and antioxidant properties," Journal of the Science of Food and Agriculture, vol. 93, no. 9, pp. 2121-2129, 2013. 